Molecular lanthanide single-ion magnets: from bulk to submonolayers

This content has been downloaded from IOPscience. Please scroll down to see the full text. 2015 J. Phys.: Condens. Matter 27183203

(http://iopscience.iop.org/0953-8984/27/18/183203)

View the table of contents for this issue, or go to the journal homepage for more

Download details:

IP Address: 128.178.174.27

This content was downloaded on 15/09/2015 at $16: 10$

Please note that terms and conditions apply. 


\title{
Molecular lanthanide single-ion magnets: from bulk to submonolayers
}

\author{
$J_{\text {Dreiser }}{ }^{1,2}$ \\ ${ }^{1}$ Ecole Polytechnique Fédérale de Lausanne, ICMP, Station 3, CH-1015 Lausanne, Switzerland \\ ${ }^{2}$ Paul Scherrer Institut, Swiss Light Source, CH-5232 Villigen PSI, Switzerland \\ E-mail: jan.dreiser@epfl.ch
}

Received 21 November 2014, revised 10 February 2015

Accepted for publication 5 March 2015

Published 20 April 2015

\begin{abstract}
Single-ion magnets (SIMs) are mononuclear molecular complexes exhibiting slow relaxation of magnetization. They are currently attracting a lot of interest because of potential applications in spintronics and quantum information processing. However, exploiting SIMs in, e.g. molecule-inorganic hybrid devices requires a fundamental understanding of the effects of molecule-substrate interactions on the SIM magnetic properties. In this review the properties of lanthanide SIMs in the bulk crystalline phase and deposited on surfaces in the (sub)monolayer regime are discussed. As a starting point trivalent lanthanide ions in a ligand field will be described, and the challenges in characterizing the ligand field are illustrated with a focus on several spectroscopic techniques which are able to give direct information on the ligand-field split energy levels. Moreover, the dominant mechanisms of magnetization relaxation in the bulk phase are discussed followed by an overview of SIMs relevant for surface deposition. Further, a short introduction will be given on x-ray absorption spectroscopy, x-ray magnetic circular dichroism and scanning tunneling microscopy. Finally, the recent experiments on surface-deposited SIMs will be reviewed, along with a discussion of future perspectives.
\end{abstract}

Keywords: single-molecule magnets, single-ion magnets, surfaces, lanthanides, magnetic anisotropy

(Some figures may appear in colour only in the online journal)

\section{Introduction}

There is currently a high level of interest in mononuclear lanthanide (Ln) complexes triggered by Ishikawa's discovery of slow relaxation of magnetization in bis(phthalocyaninato)terbium about a decade ago [1]. Previously, this phenomenon had been observed in polynuclear transition metal clusters, socalled single-molecule magnets (SMMs) [2-4]. In this context, single-ion magnets (SIMs) can be defined as mononuclear metal-organic complexes which retain their magnetization for a significant amount of time ranging from milliseconds up to several hours. While several transition metal SIMs have been demonstrated [5, 6], Ln ions are particularly good candidates for SIMs because most of them feature large spin and unquenched orbital angular momenta, and ligand-field splittings are in general larger than those of first row transition metal ions [7]. Similar to polynuclear coordination clusters SIMs are true quantum magnetic entities, that is, they exhibit a discrete spectrum of magnetic quantum states. This quantum magnetism naturally asks for an investigation whether such molecules can be used as qubits to encode and manipulate quantum information $[8,9]$. The key quantity for such applications is the transverse relaxation or decoherence time of the angular momentum characterizing on which timescale the stored quantum information decays. First demonstrations of quantum coherence in polynuclear clusters have been made [10-12], however, only very few experiments have been reported on mononuclear $4 \mathrm{f}$ systems [13-15]. 
In order to exploit the molecules' properties, e.g. in spintronics applications [16-18] it is necessary to transfer the molecules from the native molecular crystal to other environments. A promising path is to deposit the molecules on appropriate surfaces [19-23], but also, a number of breakthroughs have been achieved using SIMs anchored on carbon nanotubes (CNTs) [24, 25]. The more simple structure of SIMs which is often less fragile than that of SMMs is advantageous here, because stabibility is needed for the molecules to survive the thermal evaporation used for vacuum deposition and eventually the interaction with the surface.

The present review attempts to give an overview of the most important aspects of these fascinating nano-objects in view of organizing and addressing them on surfaces. It tries to address fundamental concepts such as ligand field, symmetries and magnetization relaxation processes as well as the most recent research on surface adsorbed SIMs. After this Introduction trivalent lanthanide ions will be discussed. Then an overview of the different relevant mechanisms responsible for magnetization relaxation in bulk SIMs will be given followed by a discussion of mainly those SIMs which currently play an important role in surface experiments. In the last section, a brief summary of x-ray absorption spectroscopy (XAS) and scanning tunneling microscopy (STM) which are useful to study surface deposited SIMs is presented, and results obtained on surface-adsorbed SIMs will be reviewed.

Note that this review does not try to list the many existing SIMs and all applicable techniques. Those topics have been subject to recently published reviews and book chapters [2629]. This Introduction will be ended with two final remarks regarding the nomenclature: While in this review SIMs are defined as organic complexes it is noted that considerable progress is made in related, but purely inorganic, systems of single metal atoms directly deposited on surfaces [30,31]. Further, it should be noted that the term SIM will be used in a wider sense, neglecting a possible further discrimination of 'field-induced' SIMs, that is, SIMs which do not show remanence but exhibit slow magnetization relaxation in an applied magnetic field.

\section{Physical properties of trivalent lanthanide ions}

\subsection{Free ion}

The free $\operatorname{Ln}(\mathrm{III})$ ion is well described by the Hamiltonian $\hat{\mathrm{H}}_{\text {free }}=\hat{\mathrm{H}}_{\mathrm{kin}}+\hat{\mathrm{H}}_{\mathrm{ee}}+\hat{\mathrm{H}}_{\mathrm{SOC}}$ including the kinetic energy, electron-electron repulsion and spin-orbit coupling (SOC) in the order of appearance of the terms. When written out, this Hamiltonian reads

$\hat{\mathrm{H}}_{\text {free }}=\sum_{i}\left(\frac{\hat{\boldsymbol{p}}_{i}^{2}}{2 m}-\frac{Z e^{2}}{r_{i}}\right)+\sum_{i<j} \frac{e^{2}}{\left|\boldsymbol{r}_{i}-\boldsymbol{r}_{j}\right|}+\sum_{i} \xi_{i} \hat{\boldsymbol{l}}_{i} \cdot \hat{\boldsymbol{s}}_{i}$.

Here, the indices $i$ and $j$ refer to the $i$ th or the $j$ th electron, respectively. $\quad \hat{\boldsymbol{p}}_{i}$ is the linear momentum operator, $m$ the electron mass, $Z$ the nuclear charge in units of $e, r_{i}$ the position vector, $\hat{l}_{i}$ and $\hat{s}_{i}$ are the orbital and spin angular momentum operators and $\xi_{i}$ the SOC strength. As a consequence of

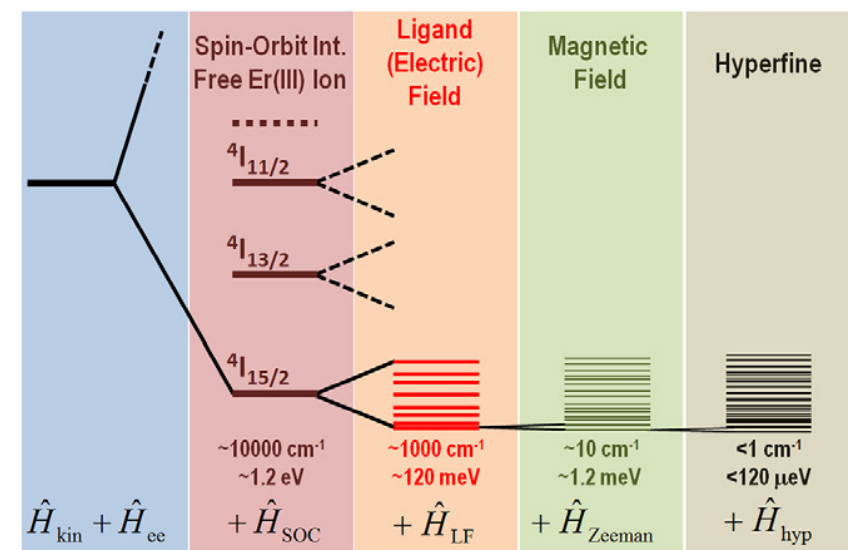

Figure 1. Energy level scheme of $\operatorname{Er}(\mathrm{III})$ depicting the effect of subsequent applications of the different Hamiltonian terms explained in the text (not to scale).

the Russell-Saunders coupling of the total spin and orbital angular momenta $S$ and $L$, Hamiltonian equation (1) gives rise to a series of multiplets characterized by the total angular momentum $J$ being a 'good' quantum number. The ground state multiplet is determined by Hund's rules. As an example, the lowest-lying multiplets of the free $\operatorname{Er}($ III) ion are depicted in figure 1. The ground state is well separated from the excited multiplets by several thousands of $\mathrm{cm}^{-1}$ (hundreds of meV), typical for the whole Ln series. Importantly, since the partially filled $4 \mathrm{f}$ shell is screened by the outer closed shells, the Ln magnetic moment is strongly localized.

\subsection{Ligand field}

Ligands are small molecules or ions which bind to the central metal ion forming a metal complex. Ligands provide spatially localized, usually negative, charges which interact via the resulting electrostatic fields with the $4 \mathrm{f}$ orbitals of the $\mathrm{Ln}(\mathrm{III})$ ions.

Because of the strong SOC in Ln compared to $3 \mathrm{~d}$ transition metals, and because of the localized nature of the partially filled 4f shell the energy scales of the electron-electron repulsion $E_{\text {ee }}$ and the SOC $E_{\mathrm{SOC}}$ are always larger than that of the ligand field $E_{\mathrm{LF}}$. This means that for Ln ions $E_{\mathrm{ee}}>E_{\mathrm{SOC}}>E_{\mathrm{LF}}$ and the ligand-field Hamiltonian $\hat{\mathrm{H}}_{\mathrm{LF}}$ which will be introduced below can be treated as a perturbation. Hence, in first order the multiplet structure of the free Ln(III) ion is not modified by $\hat{\mathrm{H}}_{\mathrm{LF}}$, and the total angular momentum $J$ remains a good quantum number. The effect of the ligand field is then to lift the degeneracy of the multiplets of electronic states which become linear combinations of the Zeeman components $\{|m\rangle\}$ (see figure 1). It should be noted that this situation corresponds exactly to the presence of magnetic anisotropy, meaning that the energy-split electronic states have different magnetic properties, for example different directions of the magnetic moment. Because of the seven $4 \mathrm{f}$ orbitals, the magnetic anisotropy 'landscape', that is, the magnetic susceptibility as a function of polar and azimuthal angles $\chi(\theta, \phi)$, can be much more complicated than in the simple axial case.

The ligand-field splittings of the multiplets are usually much larger than those obtained in $3 \mathrm{~d}$ transition metals. 
Table 1. Examples of Stevens operators.

\begin{tabular}{ll}
\hline$\hat{O}_{0}^{2}$ & $3 \hat{J}_{z}^{2}-J(J+1)$ \\
$\hat{O}_{0}^{4}$ & $35 \hat{J}_{z}^{4}-[30 J(J+1)-25] \hat{J}_{z}^{2}+3 J^{2}(J+1)^{2}-6 J(J+1)$ \\
$\hat{O}_{4}^{4}$ & $\frac{1}{2}\left[\hat{J}_{+}^{4}+\hat{J}_{-}^{4}\right]$ \\
\hline
\end{tabular}

Furthermore, the orbital angular momentum of the latter is often quenched by the ligand field while this does not happen for the $\mathrm{Ln}(\mathrm{III})$ ions giving rise to large magnetic momenta. This holds true especially for the late Ln(III) which exhibit parallel alignment of their spin and orbital angular momenta. The Gd(III) ion with ground state ${ }^{8} \mathrm{~S}_{7 / 2}$ represents a special case because of its vanishing orbital angular momentum. As a consequence, it does not interact in first order with the ligand field resulting in small ligand-field splittings and weak magnetic anisotropy.

Adding the ligand field term as well as Zeeman and hyperfine interactions to equation (1) yields

$$
\hat{\mathrm{H}}=\hat{\mathrm{H}}_{\text {free }}+\hat{\mathrm{H}}_{\mathrm{LF}}+\hat{\mathrm{H}}_{\text {Zeeman }}+\hat{\mathrm{H}}_{\text {hyp }} \text {. }
$$

The effect of these Hamiltonian terms on the energy spectrum are visible in figure 1.

In a phenomenological notation the ligand field can be expressed as $\hat{\mathrm{H}}_{\mathrm{LF}}=\sum_{i, k, q} \tilde{B}_{k}^{q} \hat{C}_{k}^{q}(i)$ ('Wybourne notation'). The $\hat{C}_{k}^{q}(i)$ are the ligand-field one-electron tensor operators acting on electron $i$ with corresponding coefficients $\tilde{B}_{k}^{q}$ and $-q \leqslant k \leqslant q, q=2,4,6$.

Since the interaction with the ligand field is small compared to the spin-orbit interaction it can be expressed in first order as Stevens operators $\hat{\mathrm{H}}_{\mathrm{LF}, \mathrm{J}}=\sum_{k, q} B_{k}^{q} \hat{O}_{k}^{q}(\boldsymbol{J})$ [32]. Here, the operators are simple functions of the components of $\boldsymbol{J}$ and their power. A few examples of relevant Stevens operators are given in table 1 . The restriction of the basis set to the ground state multiplet provides an efficient simplification in which $\hat{\mathrm{H}}_{\text {free }}$ is only implicitly contained. This is reasonable for the description of the magnetic properties but of course it fails in describing optical absorption or luminescence which have different multiplets as initial and final states. With these considerations one has

$$
\hat{\mathrm{H}}_{\mathrm{J}}=\hat{\mathrm{H}}_{\mathrm{LF}, \mathrm{J}}+\hat{\mathrm{H}}_{\text {Zeeman }, \mathrm{J}}+\hat{\mathrm{H}}_{\mathrm{hyp}, \mathrm{J}},
$$

with $\hat{\mathrm{H}}_{\mathrm{LF}, \mathrm{J}}$ as previously defined and $\hat{\mathrm{H}}_{\text {Zeeman, } \mathrm{J}}=g_{\mathrm{J}} \mu_{\mathrm{B}} \boldsymbol{J}$. $B$. The Zeeman term has been rewritten using the Landé $g$-factor which is given by $g_{\mathrm{J}}=(3 / 2)+$ $((S(S+1)-L(L+1)) / 2 J(J+1))$ and $\mu_{\mathrm{B}}$ is the Bohr magneton. The hyperfine interaction $\hat{\mathrm{H}}_{\text {hyp,J }}$ will not be discussed here in detail, and its energy scale is too small to be directly observed in most spectroscopic experiments on SIMs. However, anticipating the discussion in section 3.1, it plays a crucial role regarding the magnetization relaxation.

The different coefficients $B_{k}^{q}$ in $\hat{\mathrm{H}}_{\mathrm{LF}, \mathrm{J}}$ are related to the $\tilde{B}_{k}^{q}$ by the tabulated Stevens factors $\alpha, \beta, \gamma$. This and the relation of the coefficients with point charge approaches have been summarized in great detail, e.g. in $[33,34]$. The $\tilde{B}_{k}^{q}-B_{k}^{q}$ transformation bears important consequences for the nature of the magnetic anisotropy: Different $\operatorname{Ln}(\mathrm{III})$ ions exhibit different magnetic anisotropies when subjected to the same ligand field $[35,36]$. The changes can be as drastic as going from easy-plane to easy-axis type anisotropy upon exchanging the $\operatorname{Ln}($ III) ion [37, 38].

Neglecting the hyperfine interaction, one is left with the experimental determination of the Stevens parameters $B_{k}^{q}$ in order to understand and describe the magnetic properties of SIMs. As it will be discussed below, the ligand-field symmetry determines which of the 27 possible Stevens parameters are allowed to be non-zero. At this point it should also be mentioned that an alternative to describing the ligand field by Stevens parameters as done here is provided by the angular overlap model [39-41] in which the parameterization is performed in a chemically more intuitive way.

\subsection{Symmetries}

Time-reversal and point-group symmetries have a severe impact on the magnetic properties of the Ln ions. In the following the effect of these symmetries will be described.

2.3.1. Time-reversal symmetry. Depending on the integer or half-integer nature of $J$ there is a fundamental difference regarding the energy spectrum at zero magnetic field: In the case of half-integer $J$ it is impossible to lift all degeneracies by a purely electric field, e.g. the ligand field, without any symmetry whatsoever. Kramers theorem [42] states that there is at least a double degeneracy left. In contrast, in the presence of magnetic fields the degeneracy is fully lifted. Kramers theorem does not hold for integer- $J$ systems, i.e. the electronic states can be nondegenerate if the ligand field exhibits a low enough symmetry. The aspect of time-reversal symmetry becomes more complicated when also the nuclear spin is included: In both integer and half-integer $J$ systems it has a pronounced effect on quantum tunneling of magnetization (QTM) (see section 3.1).

2.3.2. Point-group symmetries. As already mentioned, in the absence of any ligand-field symmetry, there are 27 non-zero Stevens parameters for $\mathrm{Ln}$ ions. It is obvious that the reliable determination of this many parameters by, e.g. fitting models to experimental data is close to impossible, even when singlecrystal magnetization $M(B)$ data and magnetic susceptibility $\chi(T)$ are available. In the presence of symmetries the number of non-zero coefficients in $\hat{\mathrm{H}}_{\mathrm{LF}, \mathrm{J}}$ can be drastically reduced because those which are not invariant under the symmetry operations of the point group of the ion's site must be identically to zero. The allowed Stevens coefficients for different ligand-field symmetries are given in table 2 . The cases of other symmetries not mentioned in this table are listed in $[33,34]$. Obviously, in the presence of high symmetry, there are only few allowed coefficients to be determined facilitating the description of the magnetic properties by Hamiltonians equations (2) and (3).

\subsection{Pseudospin-1/2 Hamiltonian}

The low-lying doublet states are most relevant in SIMs since their composition and wavefunctions have a strong influence on the speed of magnetization relaxation. Usually these states 
Table 2. Allowed Stevens operator coefficients for selected ligand-field symmetries. Adapted from [34].

\begin{tabular}{|c|c|c|c|c|c|c|c|c|c|c|c|c|c|c|c|}
\hline & $B_{0}^{2}$ & $B_{ \pm 1}^{2}$ & $B_{ \pm 2}^{2}$ & $B_{0}^{4}$ & $B_{ \pm 1}^{4}$ & $B_{ \pm 2}^{4}$ & $B_{ \pm 3}^{4}$ & $B_{ \pm 4}^{4}$ & $B_{0}^{6}$ & $B_{ \pm 1}^{6}$ & $B_{ \pm 2}^{6}$ & $B_{ \pm 3}^{6}$ & $B_{ \pm 4}^{6}$ & $B_{ \pm 5}^{6}$ & $B_{ \pm 6}^{6}$ \\
\hline$C_{4}\left|S_{4}\right| C_{4 \mathrm{~h}}$ & + & & & + & & & & \pm & + & & & & \pm & & \\
\hline$D_{4}\left|C_{4 \mathrm{v}}\right| D_{2 \mathrm{~d}} \mid D_{4 \mathrm{~h}}$ & + & & & + & & & & + & + & & & & + & & \\
\hline$C_{3} \mid S_{6}$ & + & & & + & & & \pm & & + & & & \pm & & & \pm \\
\hline$D_{3}\left|C_{3 \mathrm{v}}\right| D_{3 \mathrm{~d}}$ & + & & & + & & & + & & + & & & + & & & + \\
\hline$C_{6}\left|C_{3 \mathrm{~h}}\right| C_{6 \mathrm{~h}}$ & + & & & + & & & & & + & & & & & & \pm \\
\hline$D_{6}\left|C_{6 \mathrm{v}}\right| D_{3 \mathrm{~h}} \mid D_{6 \mathrm{~h}}$ & + & & & + & & & & & + & & & & & & + \\
\hline$T_{\mathrm{d}}|O| O_{\mathrm{h}}$ & & & & + & & & & + & + & & & & + & & \\
\hline
\end{tabular}

are well separated in energy from excited states giving rise to an Ising magnet behaviour. Since mainly the lowest-lying states are involved in the SIM dynamics, one could conclude that all other states can be neglected. Section 3 reveals that this is not true, especially when magnetization relaxation is dominated by spin-phonon coupling. Furthermore, the ligand field determines the wavefunctions of the electronic states. Condensing the basis set down to two states and working with a pseudospin $\tau=1 / 2$ Hamiltonian, however, can be useful if ligand-field symmetry is too low to facilitate an experimental determination of all allowed Stevens parameter values. The number of parameters is then strongly reduced since there are no ligand-field coefficients, and $\tau=1 / 2$ can only be split by a magnetic field. The magnetic anisotropy is reintroduced via an anisotropic, effective $g$ tensor $\hat{\mathrm{H}}=\mu_{\mathrm{B}} \hat{\tau} \cdot \boldsymbol{g} \cdot \boldsymbol{B}[43,44]$. Other similar models based on a restricted but larger Hilbert space have also been successfully applied to polynuclear clusters containing lanthanides $[45,46]$.

\subsection{Determination of ligand-field parameters}

As mentioned before, in order to understand magnetic properties of SIMs it is necessary to determine ligand-field parameters, e.g. expressed as Stevens operator coefficients, from experimental data. Powder magnetization $M(B)$ and susceptibility $\chi(T)$ data are barely sufficient for multiple parameter determination. The availability of single-crystal magnetic data improves the situation, however, even in such a case the accuracy of the excited energy levels and the corresponding wave functions will be limited since at the elevated temperatures needed to populate these energy levels many other levels are populated, too, leading to an average magnetic response.

Thus the most preferred techniques, in addition to the magnetization measurements, are spectroscopic techniques such as electron paramagnetic resonance (EPR) [43], inelastic neutron scattering (INS) $[47,48]$ or optical absorption and luminescence [49-51]. Spectroscopic techniques allow for the addressing of the energies and wave functions of each multiplet substates separately because they directly probe the energetic splitting between an initial state, which is the ground state at low temperatures, and a final state.

The typical initial and final states associated with the different spectroscopic techniques are sketched in figure 2. The lowest energy scales are typically probed by EPR spectroscopy covering energies of $\sim 0.3-30 \mathrm{~cm}^{-1}(0.04-3.7 \mathrm{meV})$. This yields the effective $g$-tensor within the pseudospin-1/2 framework described earlier in the text. With high-frequency

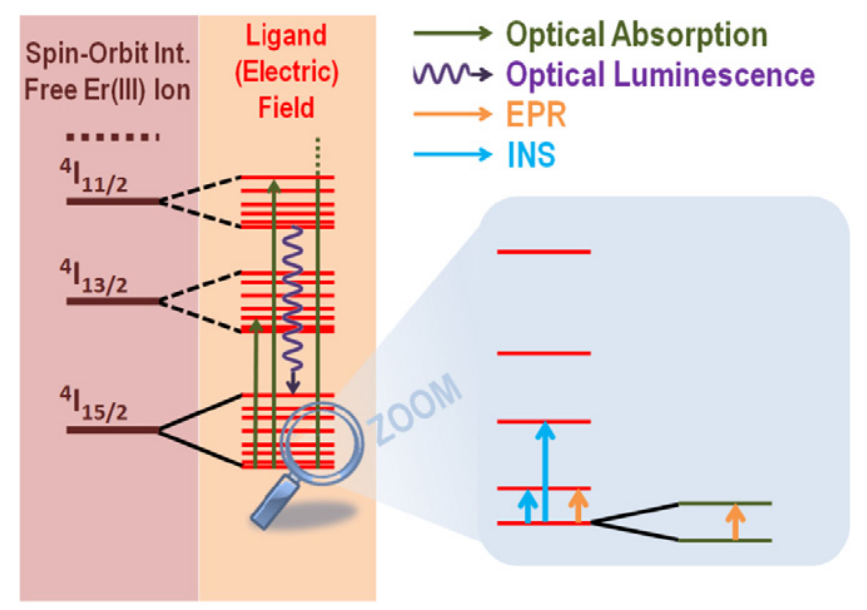

Figure 2. Energy level scheme of $\operatorname{Er}(\mathrm{III})$ depicting the typical energy levels involved in optical, neutron and magnetic resonance spectroscopic techniques.

EPR [52-57] it is often possible to measure the energy of the first excited state. However, many Ln SIMs exhibit a rather large excited-state separation which is too large even for state-of-the-art high-frequency EPR setups. INS, in which the energy loss of neutrons upon, e.g. exciting magnetic transitions in a SIM, is measured, has only been exploited rarely $[58,59]$ and earlier in an $\mathrm{Yb}$ complex [50]. It covers an energy range of up to several hundreds of $\mathrm{cm}^{-1}(\sim 50 \mathrm{meV})$, yet, this is still not enough to reach the highest energy levels of ligandfield split multiplets. High-resolution optical spectroscopy is probably the most powerful technique in this respect, however, the resulting absorption and luminescence spectra can be difficult to interpret because of the presence of vibrational bands $[51,60]$. Also the $4 \mathrm{f}-4 \mathrm{f}$ optical transitions may be dominated by strong ligand absorption bands.

Because of the many experimental obstacles there is a lot of interest in determining ligand-field parameters from first principles methods. The reason is obvious: If reliable predictions are available, promising molecular structures can be readily identified and research efforts can be focused on the most attractive candidates. It turns out, however, that such calculations are by no means an easy task. Multiconfigurational self-consistent field methods such as CASSCF, initially employed for the study of the magnetic properties of a $\mathrm{Cu}(\mathrm{II})-\mathrm{Gd}(\mathrm{III})$ complex [61], have turned out to be quite successful for the $a b$ initio calculation of magnetic properties of Ln ions [62-64]. The transverse $g$-factors $\left(g_{x x}, g_{y y}\right)$ found in the calculations provide a measure of the ground-state mixing introduced by non-axial ligand-field contributions. 
This mixing strength, in turn, indicates how prone a system is to QTM induced by intermolecular and hyperfine interaction, e.g. $[65,66]$. This indeed has been explicitly demonstrated to correlate with the SIM behavior for the case of Dy mononuclear complexes [65]. The exact energy spectrum could be obtained within an accuracy of 30\% [59].

The program CONDON [67-69] allows for a treatment of the full Hilbert space of all possible microstates of the 4f shell and outputs magnetic properties such as magnetic susceptibility and field-dependent magnetization. Similarly, the recently developed software SIMPRE [70] is able to calculate magnetic properties of SIMs from sets of Stevens parameters, and it works with effective point-charge models which can provide a more intuitive insight than full $a b$ initio calulcations. However, their performance depends crucially on the procedure of obtaining the effective coordinates and the magnitude of the effective point charges which do not have to coincide with the coordinates and the formal oxidation states of the ligand atoms. Nevertheless, effective point-charge models have been demonstrated to be useful tools when working with SIMs [36, 71-73].

\section{Relaxation of magnetization}

Magnetization relaxation in SIMs arises from the coupling of the magnetic moment to different environments such as the lattice of the molecular crystal, other neighboring SIMs in the lattice or the conduction band electrons in a metallic substrate on which SIMs are deposited. Relaxation leads to the time evolution of the SIM magnetization towards thermal equilibrium with the environment, after it has been brought outof-equilibrium by an external perturbation. The response to the perturbation is related to the fluctuations of the single magnetic moments by the fluctuation-dissipation theorem. Since in SIMs the magnetization dynamics takes place between the lowest energy Ising doublet states as already mentioned in the previous section, relatively simple models involving two quantum states (and partially a third excited state) are able to grasp the mechanisms of QTM and spin-phonon coupling.

For an angular momentum there is longitudinal and transverse relaxation, with associated timescales $\tau_{1}$ and $\tau_{2}$. The first one involves an exchange of energy with the lattice or phonon bath, while the second one does not. Here, the longitudinal relaxation is referred to as magnetization relaxation while the transverse relaxation is the decoherence, i.e. the loss of phase information. Obviously, for classical data storage applications, the decoherence is less important, while long decoherence times are of high interest for quantum computing schemes exploiting SIMs.

Magnetization relaxation in Ln salts has been a topic of interest since the 1960s [43,74-76] being relevant nowadays for mononuclear and polynuclear Ln-containing complexes [77,78]. While the mechanisms of magnetization relaxation in SIMs are at least partially understood, the knowledge about relaxation in surface-deposited SIMs is a nearly blank sheet. Yet, it is certainly a topic of high interest.

For a molecular complex to be a SIM, simply all magnetization relaxation mechanisms have to be inefficient.
At the lowest temperatures of a few Kelvin the dominant relaxation mechanism is usually hyperfine-mediated QTM, which is governed by the nature of the involved $\mathrm{Ln}$ (III) ion and its nuclear spin as well as by the ligand-field symmetry. At slightly higher temperatures, spin-phonon coupling becomes important. Spin-phonon coupling gives rise to a variety of magnetization relaxation mechanisms. The associated relaxation rates exhibit different dependencies on temperature and magnetic field. In the following the different processes relevant for magnetization relaxation in SIMs will be discussed in detail. Their distinct dependence on temperature and magnetic field, which allows to separate out which mechanism is dominant in which regime, will be given.

\subsection{Quantum tunneling of magnetization}

Since its celebrated first observation [4, 79-82] magnetization tunneling, most frequently termed as QTM, is omnipresent in the field of SMMs and SIMs, and it almost has become a nuisance because it limits the magnetization relaxation times at the lowest temperatures. The introduction to QTM presented here will be short and more details can be found in dedicated reviews or book chapters $[4,83,84]$.

In analogy to the example presented in [4], one can define the Hamiltonian of a two-state system $\{|\downarrow\rangle,|\uparrow\rangle\}$ such as for $S=1 / 2$ and $\hat{S}_{z}|\downarrow\rangle=-1 / 2|\downarrow\rangle, \hat{S}_{z}|\uparrow\rangle=+1 / 2|\uparrow\rangle$, with the matrix representation $H=\left(\begin{array}{cc}-\Delta & \hbar \omega_{T} \\ \hbar \omega_{T} & \Delta\end{array}\right)$. The energy eigenvalues of this Hamiltonian are $E_{ \pm}= \pm \sqrt{\Delta^{2}+\left(\hbar \omega_{T}\right)^{2}}$. For the imagination of the reader, the parameters $\Delta$ and $\hbar \omega_{T}$ can be identified with a longitudinal and a transverse Zeeman splitting $\Delta=g \mu_{\mathrm{B}} B_{z} \hat{S}_{z}$ and $\hbar \omega_{T}=g \mu_{\mathrm{B}} B_{x} \hat{S}_{x}$. It is further useful to call the quantity $\omega_{T}$ the tunnel frequency for reasons detailed below. Obviously, $H(\Delta)$ exhibits an energy level crossing for $\hbar \omega_{T}=0$ and an anticrossing, or avoided crossing, for $\hbar \omega_{T} \neq 0$, with a minimum splitting of $E_{+}-E_{-}=2 \hbar \omega_{T}$. It can be shown that the time evolution obtained from the Schrödinger equation is given by $|\psi(t)\rangle=x(t)|\downarrow\rangle+y(t)|\uparrow\rangle$. The functions $x(t)$ and $y(t)$ depend on the initial conditions at $t=0$ as well as on whether the states are on resonance $(\Delta=0)$ or if they are far from resonance $\left(\Delta \gg \hbar \omega_{T}\right)$. Regarding the on resonance case it is assumed that $\hbar \omega_{T}$ is smaller than the width of the energy levels. Far from resonance, $x(t)=\exp \left(-i \omega_{T} t\right)$ and $y(t)=0$, meaning that the system always remains in the $|\downarrow\rangle$ state. However, on resonance $x(t)=\cos \left(\omega_{T} t\right)$ and $y(t)= \pm \sin \left(\omega_{T} t\right)$, i.e. the system undergoes an oscillation between the two states with angular frequency $\omega_{T}$. This is exactly the tunneling phenomenon, and it has the off-diagonal terms in $H$ at its origin. Note that these solutions only represent examples for the initial condition $\psi|t=0\rangle=|\downarrow\rangle$ but they reflect nicely the basic mechanism of QTM. Importantly, this simple yet fundamental treatment can be generalized to $S>$ $1 / 2$. Furthermore, the exact physical meaning of the quantities $\Delta$ and $\hbar \omega_{T}$ depends on the system under investigation. The main point is that QTM is caused by small perturbations of any kind leading to off-diagonal elements in the Hamiltonian.

As a second example a system possessing an easy-axis magnetic anisotropy parallel to the $z$ direction is given by $\hat{\mathrm{H}}=D\left[\hat{S}_{z}^{2}-S(S+1) / 3\right]+g \mu_{\mathrm{B}} \hat{S}_{z} B_{z}+E\left(\hat{S}_{x}^{2}-\hat{S}_{y}^{2}\right)$ with $D<0$ 


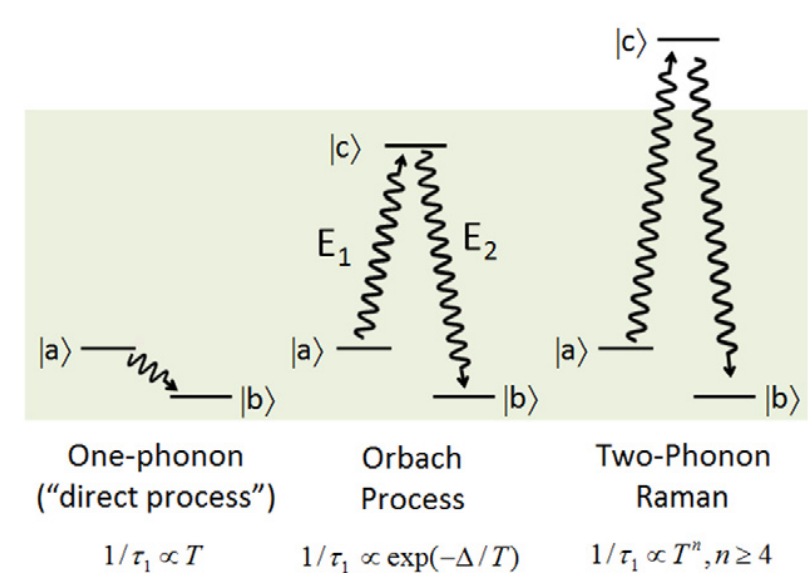

Figure 3. Most relevant magnetization relaxation mechanisms arising from spin-phonon coupling and their temperature dependence. The shaded region denotes the phonon continuum.

and initially $E=0 . D$ and $E$ are related to the previously introduced Stevens coefficients by $D=3 B_{0}^{2}$ and $E=B_{2}^{2}$. For $E=0$ its eigenstates are well described by the $m_{s}$ quantum numbers, and the states with $+m_{s}$ and $-m_{s}$ are degenerate in the absence of a magnetic field. If a weak perturbation $|E| \ll|D|$ is turned on, the eigenstates of $\hat{\mathrm{H}}$ become admixtures of different $m_{s}$ states. In the case of even number of electrons in the $4 \mathrm{f}$ shell ('non-Kramers ion'), this leads to a small energy splitting and QTM when the Zeeman energy becomes small compared to the tunnel splitting. Importantly, QTM is a resonant effect, which becomes relevant only at or in the vicinity of energy level crossings. This implies that for the $S=1 / 2$ system described before, there is only one resonance, but there are more than one in a system with larger spin.

The simple examples showed that tunneling is strongly dependent on the applied magnetic field. In SIMs it is particularly efficient at or close to zero magnetic field, which often gives rise to so-called butterfly type or waistrestricted hysteresis loops. It should be further noted that the hyperfine interaction as well as dipolar fields resulting from the interaction with neighboring magnetic ions can introduce off-diagonal terms and thus promote QTM. This can happen when the splitting between two electronic states is smaller than the maximum hyperfine splitting or the dipolar-field induced splitting, respectively. If the number of electrons in the open shell is odd ('Kramers ion'), that is, for half-integer electronic spin, there is strict double degeneracy of the electronic states because of Kramers theorem. This should, in principle, preclude QTM at $\mu_{0} H_{z}=0 \mathrm{~T}$, however, experiments on $\mathrm{LnPc}_{2}$ show that hyperfine interaction indeed promotes QTM also in the case of an odd number of electrons $[85,86]$. Finally, QTM between magnetic ground states is naturally independent of temperature. However, relaxation becomes temperature dependent when tunneling proceeds across excited states $[77,81,87]$.

\subsection{Spin-phonon interaction}

In contrast to the temperature-independent QTM, spin-phonon interaction gives rise to a number of temperature dependent relaxation mechanisms. The most relevant ones are sketched in figure 3. The Zeeman-split Ising doublet ground states between which the relaxation process takes place are denoted as states $|a\rangle$ and $|b\rangle$. State $|c\rangle$ is an excited state which is involved in the two-phonon relaxation mechanisms as described below.

3.2.1. One-phonon (direct) process. In the so-called direct process $[43,75,88]$ the relaxation from $|a\rangle$ to $|b\rangle$ takes place via the emission or absorption of a single phonon of the energy difference $E_{\text {phon }}=E_{\mathrm{a}}-E_{\mathrm{b}}$ with $E_{i}$ denoting the energy of state $|i\rangle$. It was shown that the corresponding relaxation rate, that is the inverse of the relaxation time, for Kramers ions varies as $\Gamma_{\mathrm{dir}, \mathrm{K}}=1 / \tau_{1} \propto H^{5} \operatorname{coth}\left(g \mu_{\mathrm{B}} \mu_{0} H / 2 k_{\mathrm{B}} T\right) \approx$ $H^{4} T$ and for non-Kramers ions as $\Gamma_{\text {dir,NK }}=1 / \tau_{1} \propto$ $H^{3} \operatorname{coth}\left(g \mu_{\mathrm{B}} \mu_{0} H / 2 k_{\mathrm{B}} T\right) \approx H^{2} T$, respectively. Here, $g$ is the ion's $g$-factor, $\mu_{\mathrm{B}}$ is the Bohr magneton, $T$ is the temperature, $H$ is the applied field and $k_{\mathrm{B}}$ is the Boltzmann constant. The last approximations are valid if the Zeeman splitting is much smaller than the thermal energy $g \mu_{\mathrm{B}} \mu_{0} H \ll 2 k_{\mathrm{B}} T$ for both Kramers and non-Kramers cases.

3.2.2. Two-phonon processes. The one-phonon process described before is inefficient because the applicable phonon density of states is small at the typical energies of ground state Zeeman splittings. Therefore, two-phonon processes involving phonons of much larger energy and larger densities of states can be more efficient in certain temperature ranges. The most important two-phonon processes are Orbach [89] and Raman $[43,75]$ processes. In both cases a phonon of energy $E_{1}$ is absorbed and another phonon of energy $E_{2}$ is emitted with the energy difference $E_{1}-E_{2}$ equal to the Zeeman splitting. The two processes differ in the energies of the involved phonons: While in the case of an Orbach process the phonons are below the maximum phonon energy, the Raman process corresponds to an absorption and reemission of virtual phonons above that energy. Raman and Orbach processes can be distinguished by their characteristic temperature dependence. In the case of the Orbach process the magnetization relaxation rate depends exponentially on the temperature:

$\Gamma_{\text {Orb }}=1 / \tau_{1} \propto \Delta^{3} \exp \left[-\Delta /\left(k_{\mathrm{B}} T\right)\right]$ for $\Delta \gg T$.

Here, $\Delta \sim E_{1} \sim E_{2}$ is the energy barrier that has to be overcome to reverse the magnetization. The dependences of Raman processes on temperature and magnetic field are given by $[43,75]$.

$$
\begin{gathered}
\Gamma_{\mathrm{Ram}, \mathrm{K}}=1 / \tau_{1}=R_{r} T^{9}+\alpha_{H} R_{r}^{\prime} T^{7} H^{2}, \\
\Gamma_{\mathrm{Ram}, \mathrm{NK}}=1 / \tau_{1}=R_{r} T^{7} .
\end{gathered}
$$

Here, $R_{r}$ and $R_{r}^{\prime}$ are material specific parameters, and $\alpha_{H}$ is a constant. Equations $(5 a)$ and $(5 b)$ hold for Kramers ions and non-Kramers ions, respectively (see section 2.3). Note that the low-lying energy level structure has a crucial influence on the relaxation dynamics, and if there are several energy levels close to the ground state the exponent in the temperature dependence of equations (5) can be significantly lower [90-92]. 

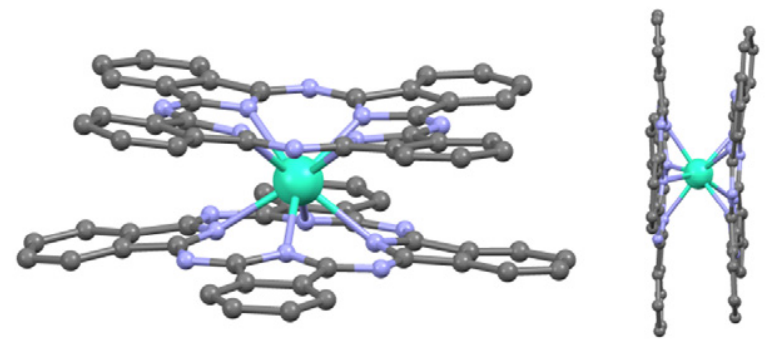

Figure 4. Molecular structure of the $\mathrm{TbPc}_{2} \mathrm{SIM}$. (Left) Oblique view; (right) side view. Color code: terbium, turquoise; carbon, gray; nitrogen, blue. Hydrogen atoms have been omitted.

\section{SIMs in the bulk phase}

It is important to achieve a deep understanding of the static and dynamic magnetic properties of SIMs in the bulk crystalline phase in order to understand the effect of the surface deposition. In this Chapter some surface-relevant SIMs will be described. As it will be discussed later, it is of interest to sublime SIMs in ultrahigh vacuum (UHV). Therefore, this review highlights the few species which have been shown to be sublimable. Those can be considered as model systems useful to study the on-surface magnetic properties of this interesting class of magnetic systems. At the end of this Chapter, some additional systems will be discussed which may be of future interest.

\section{1. $L n P c_{2}$}

This class of mononuclear $\mathrm{Ln}(\mathrm{III})\left[\mathrm{LnPc}_{2}\right]^{-} \mathrm{TBA}^{+}$(with $\left.\mathrm{TBA}^{+}=\mathrm{N}\left(\mathrm{C}_{4} \mathrm{H}_{9}\right)_{4}^{+}\right)$complexes is currently attracting a lot of interest. Here, a $\operatorname{Ln}(\mathrm{III})$ ion is sandwiched ('double decker' structure) between two phthalocyaninato dianions (Pc) giving rise to an eight-coordinated ligand field. The skew or twist angle between the Pc sheets and thus the symmetry of the ligand field depends crucially on the central metal ion. For the earlier lanthanides it is close to $0^{\circ}$, while for the late lanthanides it is close to $45^{\circ}$ [93] in the latter case corresponding to a square antiprism with $D_{4 \mathrm{~d}}$ symmetry.

Most studies are focused on the $\mathrm{Tb}(\mathrm{III})$ congener because of its particularly slow relaxation of magnetization. It has been shown that $\left[\mathrm{TbPc}_{2}\right]$ exists in three different oxidation states as anion $\left[\mathrm{TbPc}_{2}\right]^{-}$, cation $\left[\mathrm{TbPc}_{2}\right]^{+}$and in neutral form $\left[\mathrm{TbPc}_{2}\right]^{0}$ with the structure of the latter depicted in figure 4. They exhibit small differences of their structures associated with slightly different dynamic magnetic properties $[94,95]$. For a fluorinated species even the doubly negatively charged complex has been demonstrated [96]. It should be noted that because of the ligand oxidation the neutral species hosts one unpaired $\pi$ electron delocalized over the two Pc ligands giving rise to an organic radical with $S=1 / 2$. Hence, counting also the Tb electronic spin of $J=6$ and the nuclear $\operatorname{spin} I=3 / 2$, the $\mathrm{TbPc}_{2}^{0}$ molecule is actually a coupled three-spin system. It was found from temperature dependent magnetic behavior of bulk material, that in $\operatorname{TbPc}_{2}^{0}$ the $J$ and $S$ are antiferromagnetically coupled $[97,98]$.

At very low temperatures $(T \sim 2 \mathrm{~K})$ the magnetization relaxation becomes slow enough such that a magnetic

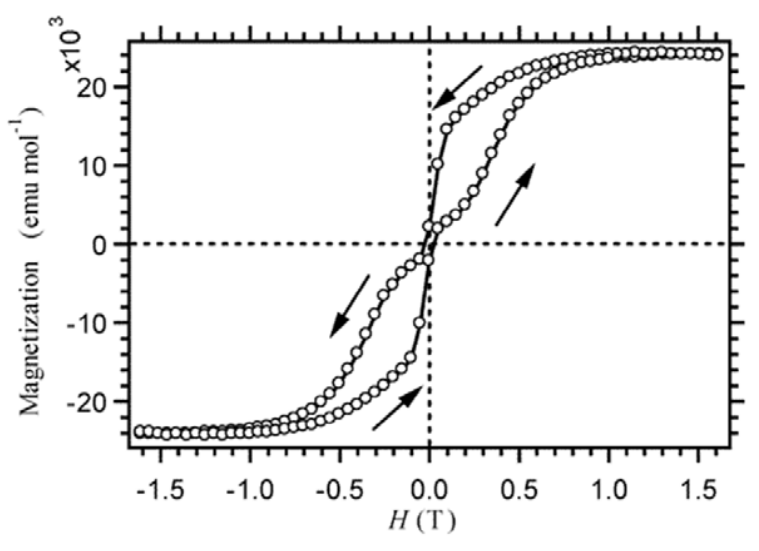

Figure 5. Magnetic hysteresis at $T=1.7 \mathrm{~K}$ of $\left[\mathrm{TbPc}_{2}\right]^{-} \mathrm{TBA}^{+}$ (with $\left.\mathrm{TBA}^{+}=\mathrm{N}\left(\mathrm{C}_{4} \mathrm{H}_{9}\right)_{4}^{+}\right)$diluted in the diamagnetic $\left[\mathrm{YPc}_{2}\right]^{-}$ $\mathrm{TBA}^{+}$. Reprinted with permission from [103]. Copyright (2004) American Chemical Society.

hysteresis can be detected in a SQUID magnetometer measurement as shown in figure 5 for the anionic complex. At these low temperatures the magnetization relaxation is determined by QTM $[99,100]$ which has been demonstrated to be driven by hyperfine interaction [85]. It was demonstrated that in $\mathrm{TbPc}_{2}^{-}$the direct process (see section 3.2) dominates the magnetization relaxation in the intermediate temperature range of 12-20 K [101]. At slightly elevated temperatures of $\sim 30 \mathrm{~K}$ it was observed that the neutral complex exhibits a slower magnetization relaxation than the anionic form [94] which was later attributed to an increase of the ligand-field strength because of the removal of one electron from the antibonding highest occupied molecular orbital (HOMO) [102]. Above that temperature, starting from $\sim 50 \mathrm{~K}$ muon spin relaxation and nuclear magnetic resonance experiments revealed that the magnetization relaxation in $\mathrm{TbPc}_{2}^{-}$and $\mathrm{TbPc}_{2}^{0}$ is thermally activated, i.e. dominated by an Orbach process (see section 3.2) with effective barriers of around $600 \mathrm{~cm}^{-1}$.

The ligand-field parameters of the $\mathrm{LnPc}_{2}^{-}$series were determined by Ishikawa et al by studying the paramagnetic shifts in nuclear magnetic resonance spectra, taking into account that the ligand-field parameters vary in a linear fashion across the lanthanide series [37, 104]. This yielded the energy spectra of the $\mathrm{LnPc}_{2}^{-}$series shown in figure 6 indicating that the $\mathrm{TbPc}_{2}^{-}$anion has the largest separation between the ground and first excited state of more than $400 \mathrm{~cm}^{-1}$. The ground state is characterized by the maximum projection $\left|J_{z}\right|=6$ corresponding to a strong easy-axis magnetic anisotropy. These findings are in line with the very long magnetization relaxation times of $\mathrm{TbPc}_{2}^{-}$. The energy level diagram also nicely illustrates the very different magnetic properties of different $\mathrm{Ln}$ (III) ions in the same ligand field (see section 2.2). A very recent spectroscopic investigation [59] is in good agreement with Ishikawa's prediction for $\mathrm{DyPc}_{2}^{-}$however, it put forward some corrections to the spectra of $\mathrm{HoPc}_{2}^{-}$and $\mathrm{TbPc}_{2}^{-}$.

Because of the high symmetry of the $\mathrm{TbPc}_{2}$ family, QTM is very slow, and it should be completely suppressed in the case of perfect $D_{4 \mathrm{~d}}$ symmetry. It was demonstrated that strong changes occur in the appearance of the hysteresis openings 


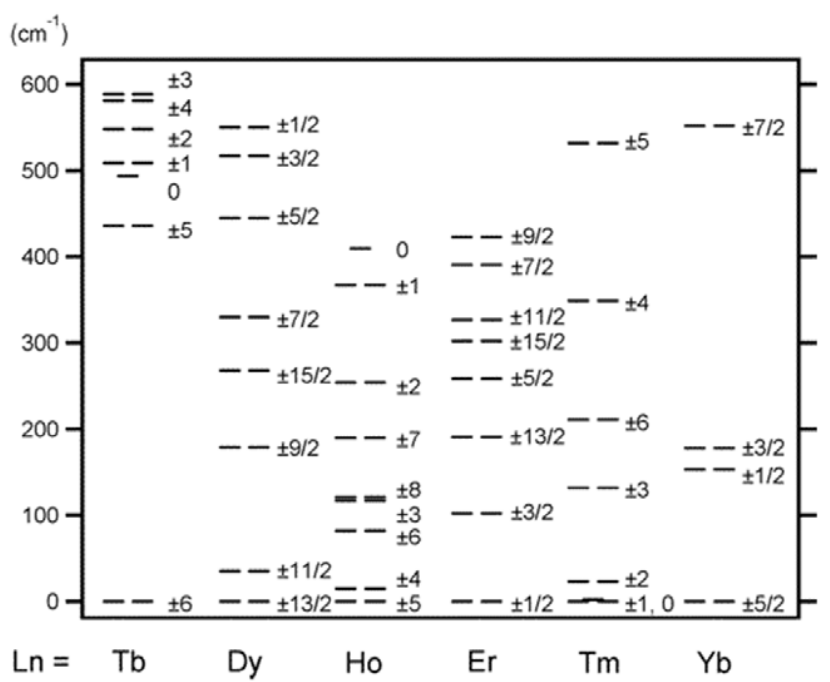

Figure 6. Energy spectra of various $\mathrm{LnPc}_{2}^{-} \mathrm{TBA}^{+}$. Reprinted with permission from [103]. Copyright (2004) American Chemical Society.

of $\mathrm{TbPc}_{2}^{-}$upon heating and sublimation [105] which were assigned to small structural diffences because of, e.g. a change in packing of molecules.

Finally, it should be noted that related Ln doubledecker complexes were also obtained employing ligands other than Pc, namely tetraphenylporphyrin [106] and octaethylporphyrin [107].

\subsection{Ln(trensal)}

Another family of Ln SIMs which has been studied in detail is the $\mathrm{Ln}$ (trensal) series, with $\mathrm{H}_{3}$ trensal $=2,2^{\prime}, 2^{\prime \prime}$ tris(salicylideneimino)triethylamine. Its structure is shown in figure 7.

Originally synthesized by Kanesato and Yokoyama [109], thorough studies of the ligand field and $4 \mathrm{f}$ electronic structures of the $\mathrm{Ln}$ ions across the $\mathrm{Ln}$ (trensal) series were undertaken by Flanagan et al $[108,110]$. The Ln(trensal) systems are excellent model systems because their $4 \mathrm{f}-4 \mathrm{f}$ transitions in the visible to near infrared optical range are accessible in contrast to the $\mathrm{LnPc}_{2}$ double deckers described before. The $4 \mathrm{f}-4 \mathrm{f}$ optical transitions are a reliable direct probe of the energy levels in ligand-field split multiplets of the $\mathrm{Ln}^{\text {III }}$ ions (see section 2.5), whereas in the $\mathrm{LnPc}_{2}$ case the $4 \mathrm{f}-$ 4f transitions are masked by strong ligand absorption. The lowest energy levels of Er(trensal) were recently characterized by EPR and INS spectroscopy [58,111]. The recent spectroscopic studies of the Ln(trensal) systems revealed that upon functionalization in the remote part of the ligand shell drastic changes in the magnetic properties can occur, as depicted in figure 8 . Yet the first coordination spheres of the pristine and functionalized compounds are nearly identical as can be seen from the structural overlays shown in figure 8. Interestingly, both Er(trensal) and Dy(trensal) exhibit slow relaxation of magnetization, while they are easyaxis and easy-plane anisotropic systems, respectively. This was attributed to barrier-independent (non-Orbach) relaxation processes [58,111]. This is important since it suggests that in such a case maximizing the magnetic anisotropy may not lead to longer magnetization relaxation times. The drastic changes in the SIM spectroscopic and magnetic properties upon the functionalization in the ligand periphery suggest that the deposition on strongly interacting surfaces will have a similar effect.

Regarding the surface deposition, the $\operatorname{Ln}$ (trensal) complexes have been shown to be sublimable (see section 5.5). Their three-dimensional trigonal pyramidal structure, very different from the $\mathrm{TbPc}_{2}$ molecules, could allow for a stronger decoupling from the surface, weaker magnetic exchange coupling to ferromagnetic surfaces (see section 5.5) and for non-flat molecular architectures.

\subsection{Endohedral metallofullerenes}

Ln-containing endohedral metallofullerenes [112,113] are a special class of SIMs. Here, a small metal nitride molecule including three trivalent metal atoms is encapsulated in a $\mathrm{C}_{80}$ cage. The $\mathrm{C}_{80}$ cage carries a formal 6 -fold negative charge, and it is thus diamagnetic, while the nitride molecule has the corresponding positive charge. Almost all Ln and some other, e.g. $3 \mathrm{~d}$ metals, can be incorporated. In contrast to all other SIMs discussed in this review, endohedral metallofullerenes are not synthesized via conventional coordination chemistry approaches but by an arc discharge technique [113]. Slow relaxation in the endohedral metallofullerenes DySc $\mathrm{N}_{2} \mathrm{~N} \mathrm{C}_{80}$ and $\mathrm{HoSc}_{2} \mathrm{~N} @ \mathrm{C}_{80}$ has been reported [114-117], with magnetization relaxation times of $\mathrm{DySc}_{2} \mathrm{~N} @ \mathrm{C}_{80}$ exceeding several hours at $2 \mathrm{~K}$. Since these molecules can be sublimed in UHV $[118,119]$ they are appealing candidates for surface studies alike the other two families of SIMs mentioned before. The molecular structure obtained from density-functional theory (DFT) geometry optimization is shown in figure 9 along with the magnetic hysteresis observed on DySc ${ }_{2} \mathrm{~N} @ \mathrm{C}_{80}$. Recent CASSCF calculations suggested that the short Dy-N bond gives rise to a very strong axial ligand field responsible for the slow relaxation of magnetization $[66,120]$.

\subsection{Other systems}

It can be very enlightening to study SIMs of different nature even if the structure leaves only little hope for the necessary stability to sustain thermal sublimation. The polyoxometalate $\mathrm{SIM}\left[\mathrm{ErW}_{10} \mathrm{O}_{36}\right]^{9-}$ was reported in $2008[38,121]$, and its $\mathrm{Gd}$ congener has been used to demonstrate the possibility of Rabi oscillations [14]. These are a first step towards full coherent control or to achieve coherent manipulation of the electronic angular momentum and, hence, identify it as a qubit candidate besides the pioneering work on manipulatig the nuclear spin in $\mathrm{TbPc}_{2}$ [15].

A further series of Ln sandwich complexes partially showing SIM behavior was demonstrated recently. Here, the $\mathrm{Ln}$ ion is placed between pentamethylcyclopentadiene $\left(\mathrm{Cp}^{*}\right)$ and $\mathrm{COT}_{2}^{-}$rings [122-124]. Moreover, an attractive class of SIMs exhibiting remarkable magnetic behavior are the sandwich-type complexes including two cyclooctatetraene dianions (COT) as ligands. While the $\mathrm{Er}(\mathrm{COT})_{2}^{-}$complex was reported in 2007 [125], the opening of a magnetic hysteresis 

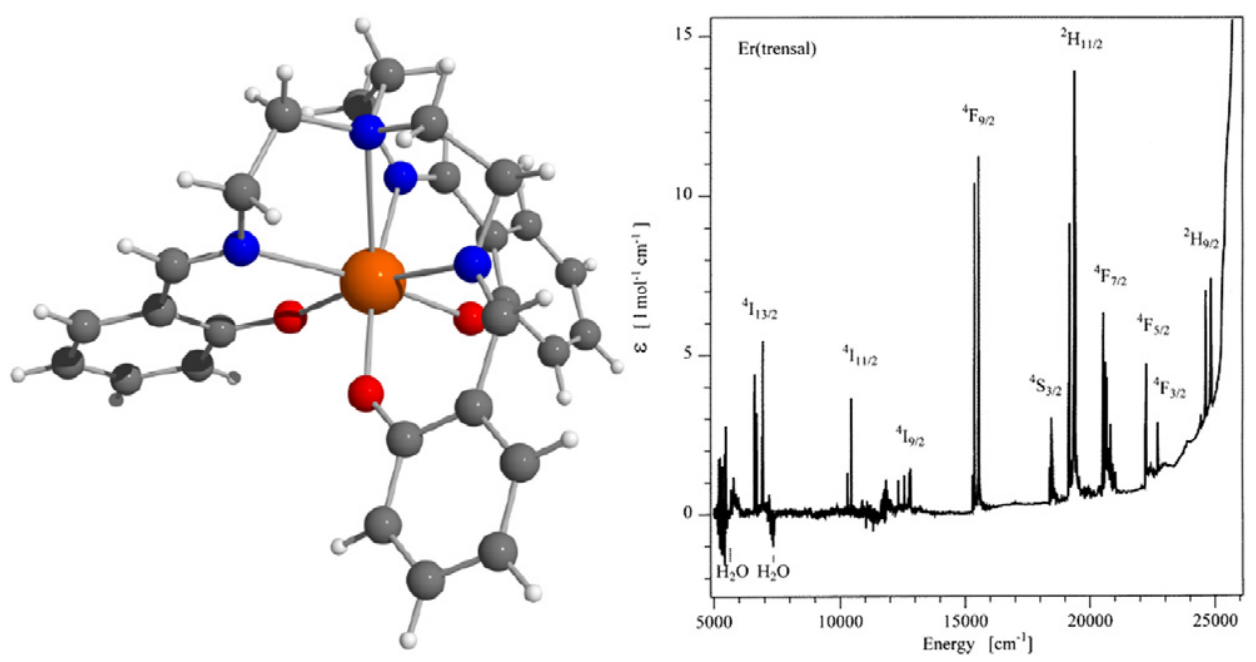

Figure 7. (Left) Molecular structure of Ln(trensal). Color code: erbium, orange; nitrogen, blue; oxygen, red; carbon, gray; hydrogen, white. (Right) Optical absorption spectrum of Er(trensal). Reprinted with permission from [108]. Copyright (2001) American Chemical Society.
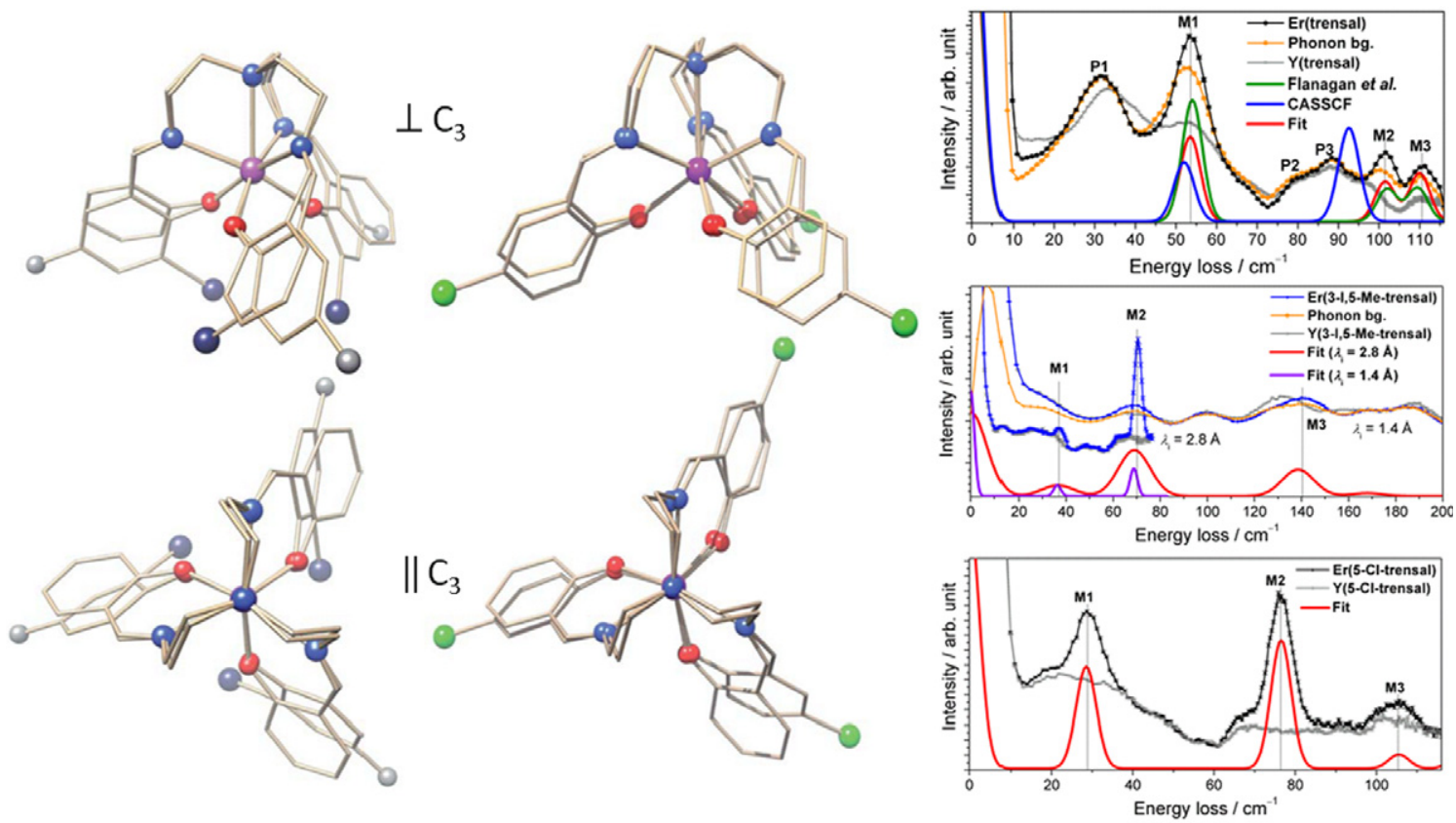

Figure 8. Overlays of Er(trensal) with $\operatorname{Er}(3-\mathrm{I}, 5-\mathrm{Me}$-trensal) (left) and with $\operatorname{Er}$ (5-Cl-trensal) (center), visualizing the differences in molecular structures. (Right) INS spectra of Er(trensal), Er(3-I,5-Me-trensal), Er(5-Cl-trensal), along with spectra of the diamagnetic yttrium analogues and the estimated phonon backgrounds revealing peaks of magnetic origin. Adapted from [58]—published by The Royal Society of Chemistry.

at remarkably high temperatures up to $10 \mathrm{~K}$ was recently demonstrated on structurally similar complexes [126-128] crystallized with different counter ions.

\section{SIMs on surfaces}

There is currently a great deal of interest in the surface deposition of organic molecules and biomolecules on surfaces. These studies are both fundamentally and technologically motivated by the observation that by using such complex adsorbates new regimes and a great diversity of self-assembled structures with interesting properties can be realized. This is of relevance in many applications in information technology, catalysis, sensing, etc. The surface studies of SIMs are probably most related to information processing or storage applications, but at this stage it is difficult to judge which other opportunities may arise in the near future. In this Chapter, the interactions relevant for the organization of SIMs on surfaces will be discussed followed by a brief introduction of XAS, $\mathrm{x}$-ray magnetic circular dichroism (XMCD) and STM which are relevant techniques for the study of SIMs on surfaces. Then, the recent results gathered on surface-deposited SIMs will be discussed.

\subsection{Molecules, surfaces and interactions}

In order to understand the behaviour of surface-deposited molecules, it is important to look at the relevant interactions 

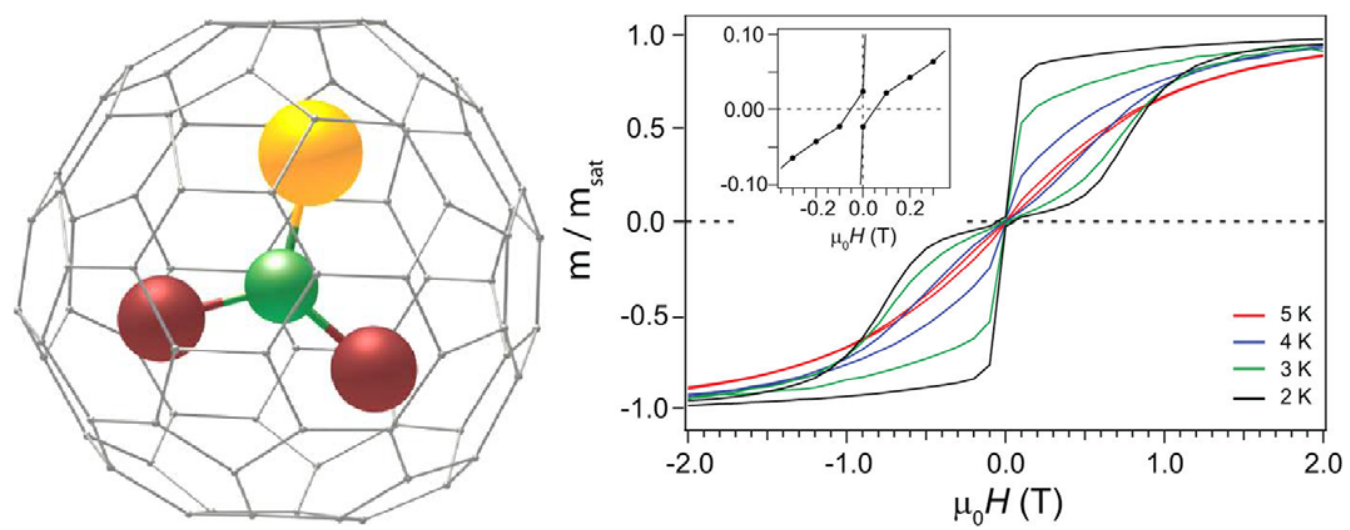

Figure 9. (Left) Structure of the endohedral metallofullerene SIM DySc $\mathrm{S}_{2} \mathrm{~N} @ \mathrm{C}_{80}$. Color code: Dy, yellow; Sc, red; green: N. The $\mathrm{C}_{80}$ cage is shown as a gray wireframe. (Right) Temperature-dependent hysteresis loops of $\mathrm{DySc}_{2} \mathrm{~N} @ \mathrm{C}_{80}$ recorded by SQUID magnetometry. Adapted with permission from [114]. Copyright (2012) American Chemical Society.
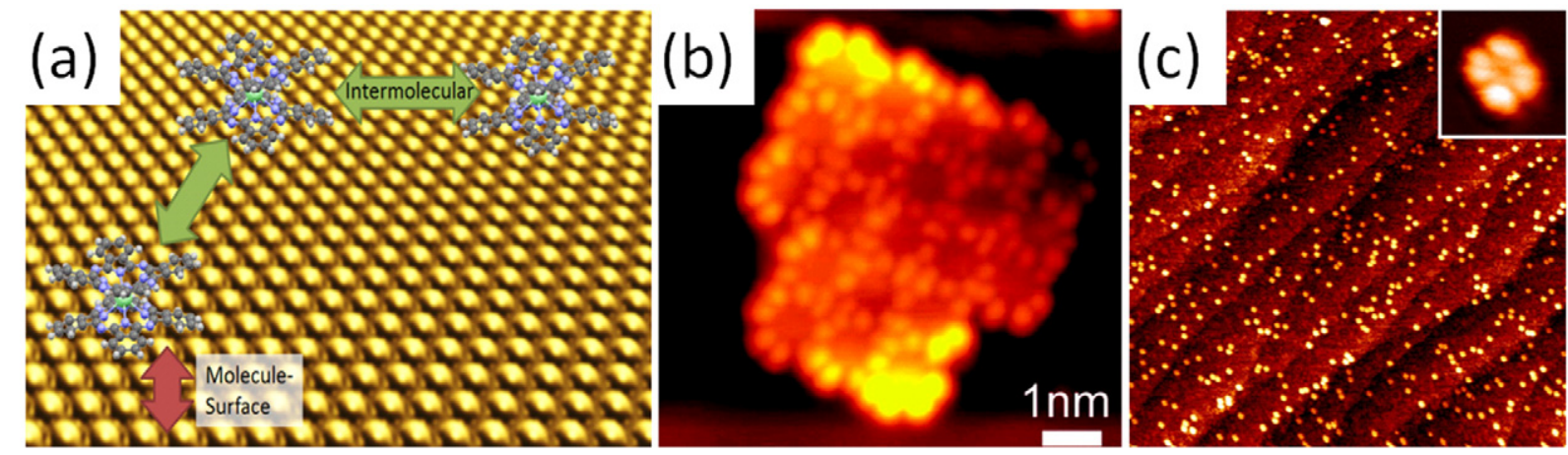

Figure 10. (a) Relevant interactions. (b) $\mathrm{TbPc}_{2}$ deposited on $\mathrm{Au}(111)$. (c) $\mathrm{TbPc}_{2}$ deposited on $\mathrm{Cu}(100), 200 \times 200 \mathrm{~nm}^{2}$. Adapted with permission from $(b)$ [131] and $(c)$ [132]. Copyright $(2009,2010)$ American Chemical Society.

that govern the adsorption of the molecules on the surface. Since the adsorption of organic molecules on a surface is an extended research topic by itself a comprehensive discussion is beyond the scope of this work, and the reader is referred to dedicated reviews, as e.g. [129, 130]. Importantly, interactions can be classified through the way they are effective into intermolecular interactions and molecule-substrate interactions, as depicted in figure 10(a). Among the former ones are hydrogen bonding, metal-ligand interactions and interactions between delocalized electron systems. Molecule-substrate interactions comprise charge transfer and covalent interaction, electric dipolar interactions of static and dynamic (van der Waals) nature and mirror charge(s). Adsorption can be weak (physisorption) or strong (chemisorption). Further, energy barriers for surface migration and rotation can play a role. The delicate balance of these interactions determines whether, e.g. self-assembled structures are formed or whether the molecules are adsorbed one-by-one at random sites.

The substrate surface certainly plays an important role as together with the molecular properties it determines the adsorption energy, migration and rotation barriers of molecules. Largely inert noble metal surfaces most often result in low adsorption energy, low migration and rotational barriers and in consequence the realization of the physisorption case. This is in contrast to more reactive transition metal surfaces which tend to form covalent bonds, or hybridize, with the adsorbates. Strong hybridization or covalent bonding is most often accompanied by the formation of sizeable migration barriers such that the molecules become immobile on the surface even at room temperature. Two example cases are shown in figure 10 where $\mathrm{TbPc}_{2}$ on $\mathrm{Au}\left(\begin{array}{lll}1 & 1 & 1\end{array}\right)$ are mobile enough to form patches of ordered molecules $(b)$ while on $\mathrm{Cu}\left(\begin{array}{ll}1 & 0\end{array}\right)$ the molecules are strongly attached to the surface $(c)$.

There are considerably less studies on semiconducting or oxide surfaces. These surfaces have a more complex structure, and they are more difficult to prepare and to image than metal surfaces. Most of the 'classical' surface science studies employing small inorganic or organic molecules are performed on $\mathrm{Si}\left(\begin{array}{lll}1 & 0 & 0\end{array}\right)$ [133-135] or $\mathrm{TiO}_{2}\left(\begin{array}{lll}1 & 1 & 0\end{array}\right)$ surfaces [136, 137].

The preparation of high-quality surfaces and the study of molecular adsorbates in UHV requires a considerable pool of equipment. Furthermore, the in-situ deposition of molecules by sublimation in UHV puts strong constraints on the thermal stability of the SIMs. Nevertheless, UHV surface preparation and in-situ molecule deposition allow that the surface is free of adsorbates prior to the molecule deposition which is of crucial importance when studying the influence of molecule-surface interactions which arise from the direct contact between molecules and the substrate. These interactions can be weaker or completely absent if (unwanted) adsorbates are present. Wet chemistry type approaches have been demonstrated to be very successful to anchor molecular magnetic clusters, SMMs and SIMs to a variety of surfaces [21,138-141]. This has 
proven to be very useful to decouple and isolate SMMs and SIMs from the surface, however, achieving direct moleculesurface electronic and magnetic coupling on purpose is rather challenging with this approach, with some few exceptions of rather inert surfaces such as $\mathrm{Au}(111)$ or highly oriented pyrolitic graphite (HOPG). Electrospraying is a promising path to the deposition of molecules while keeping the substrate free from adsorbates. More details about structuring and depositing molecules on surfaces can be found in dedicated reviews $[19,20]$.

A topic that has been largely neglected in the field of SIMs is that the structure of surface-adsorbed molecules does not have to be identical to that in the bulk crystalline phase. There are several reasons for that: Simply, there could be damage to the molecules during the sublimation process, or modifications can be induced by, e.g. charge transfer, or distortions and symmetry breaking occur because of the molecule-surface interactions quoted above. This issue should not be neglected when dealing with surface-adsorbed SIMs, and it will be discussed more specifically in the sections below reviewing the experiments on the different adsorbed species of SIMs. It is noted that the on-surface experimental study of changes of the Ln electronic structure because of distortions of the molecular structure or because of charge transfer and molecule-surface hybridization is very challenging. The study of the lowest electronic states by, e.g. inelastic STM spectroscopy mentioned below or other applicable techniques could deliver some insight here.

\subsection{X-ray absorption spectroscopy}

$\mathrm{XAS}$ and XMCD in the soft $\mathrm{X}$-ray range (photon energies of $\sim 0.1-5 \mathrm{keV}$ ) [142-144] are very powerful techniques to investigate the magnetic properties of surface-deposited SIMs and SMMs in the sub- and few monolayer range. XMCD is element specific because the magnetic signal from the element under consideration can be strongly increased with respect to the unwanted background. The absorption cross sections are very large in the soft $\mathrm{x}$-ray range, resulting in ultrahigh sensitivity of ca. $10^{11}$ molecules which is far superior to that of a state-of-the-art SQUID magnetometer. Moreover, XAS in total electron yield (TEY) mode is strongly surface sensitive and sum rules $[145,146]$ allow for the extraction of absolute values of spin and orbital magnetic moments.

XMCD refers to the difference between two absorption spectra recorded with the opposite circular polarizations of the incident $\mathrm{x}$-rays, i.e. $I_{\mathrm{XMCD}}\left(\hbar w_{\mathrm{ph}}\right)=I_{+}\left(\hbar w_{\mathrm{ph}}\right)-I_{-}\left(\hbar w_{\mathrm{ph}}\right)$ with $\hbar w_{\mathrm{ph}}=E_{\mathrm{ph}}$ the photon energy. The strength and sign of the integrated XMCD spectrum is proportional to the projection of the element-specific magnetic moment $\mathbf{M}$ onto the beam propagation direction $\mathcal{S}$, i.e. $\int I_{\mathrm{XMCD}} \mathrm{d} E_{\mathrm{ph}} \propto M \cdot \mathcal{S}$. To rationalize the XMCD effect in the atomic-like lanthanides, it is highly instructive to consider the simple case of the $\mathrm{Yb}(\mathrm{III})$ ion depicted in figure 11 which was treated already in the early days of XMCD by Goedkoop et al [147]. At the $\mathrm{Yb} \mathrm{M}_{5}$ edge an electron is excited from the filled $3 d_{5 / 2}$ core levels to the open $4 \mathrm{f}$ shell. Hence, the initial and final configurations are $3 d^{10} 4 f^{13}$ and $3 d^{9} 4 f^{14}$, respectively, giving rise to ${ }^{2} F_{7 / 2}$ and

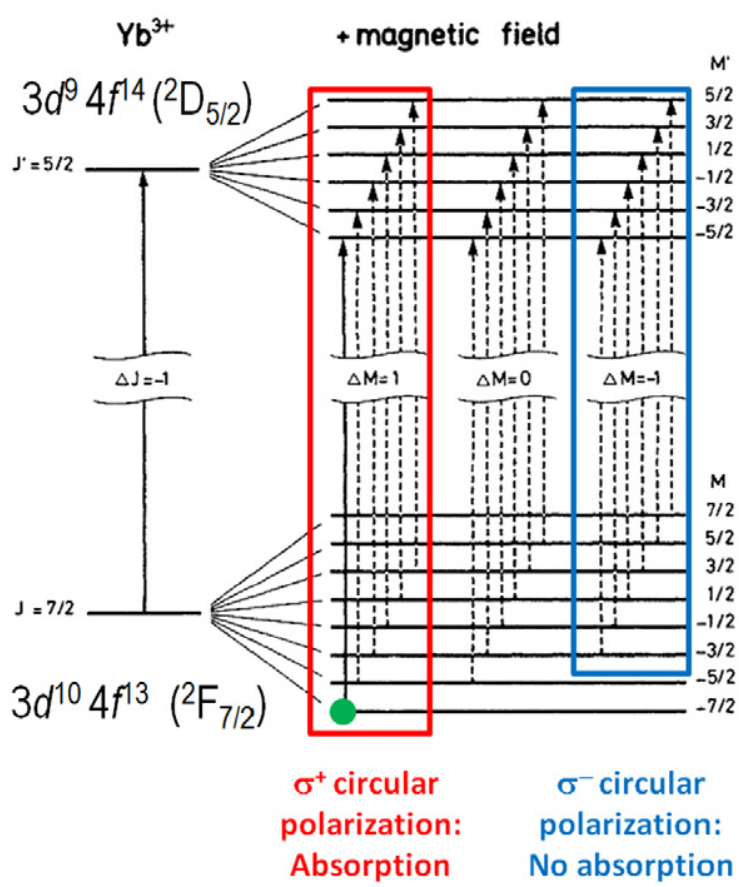

Figure 11. Origin of the XMCD effect in $\mathrm{Yb}(\mathrm{III})$. Adapted figure with permission from [147]. Copyright (1988) by the American Physical Society.

${ }^{2} \mathrm{D}_{5 / 2}$ ground state multiplets. According to the selection rules for electric dipole transitions, the transition between these multiplets is allowed with $J^{\prime}-J=\Delta J=-1$. By choosing the circular polarizations of the light, the transitions with either $M^{\prime}-M=\Delta M=+1$ or $\Delta M=-1$ are selected. Here $J, M$ and $J^{\prime}, M^{\prime}$ are the quantum numbers of the total angular momentum and its $z$-projection of the initial and final states, respectively. In the extreme case where only the $|M=-7 / 2\rangle$ state is populated which can be realized in a strong magnetic field at low temperatures, this leads to the presence of absorption with $\Delta M=+1$, i.e. for $\sigma^{+}$ circularly polarized light and the absence of absorption for $\Delta M=-1$, i.e. $\sigma^{-}$circularly polarized light as depicted in figure 11 by red and blue boxes. The relationship of the absorption cross sections for the different $\mid M>$ states is given by the Wigner- $3 j$ symbol, $\left(\begin{array}{ccc}J & 1 & J^{\prime} \\ M & m & -M^{\prime}\end{array}\right)^{2}$. The parameter $m$ refers to the polarization of the light where $m= \pm 1$ for the two circular polarizations. Since the magnetic moment of the studied $\operatorname{Ln}(\mathrm{III})$ ion is determined by the occupation of $|M\rangle$ states or their superpositions, it is mapped into the XMCD via the polarization-dependent absorption.

Technically, the soft-XAS is often measured in TEY mode, i.e. the current originating from electrons emitted from the sample by the X-rays is measured. Since the electron escape depth is only a few nanometers, the TEY signal is strongly surface sensitive. Because of the very large absorption cross sections in the soft $\mathrm{x}$-ray regime the XAS and XMCD experiments are usually performed in UHV environment. The need for energy and polarization-tunable x-rays requires a synchrotron as $\mathrm{x}$-ray source. There are a few beam lines specialized in high-field, low-temperature XMCD and which are equipped with in-situ surface-science tools necessary for 


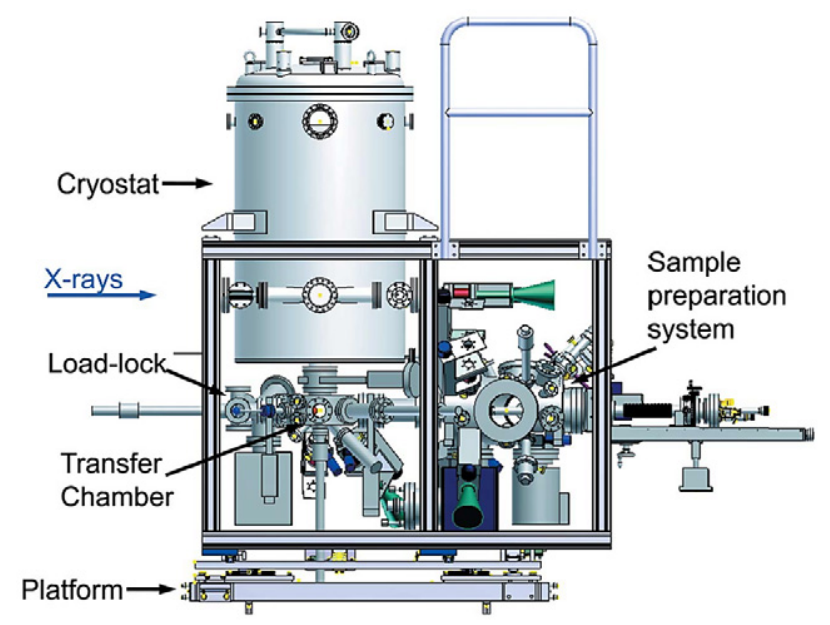

Figure 12. Preparation and measurement chambers (Cryostat) at the $\mathrm{X}$-Treme beam line at the Swiss Light Source. Reproduced from [148] with permission from the International Union of Crystallography.

the preparation of surface-adsorbed molecule samples. In figure 12 the vacuum chamber system of the X-Treme beam line [148] at the Swiss Light Source, Paul Scherrer Institut, is shown. Besides a fast entry lock and a transfer chamber, this system includes the measurement chamber, a preparation chamber and an STM chamber. Similar equipment exists e.g. at the ID08 (now ID32) beam line at European Synchrotron Radiation Facility (ESRF) and at the DEIMOS beam line at SOLEIL [149].

\subsection{Scanning tunneling microscopy}

In an STM, an atomically sharp tip is scanned over the surface of a sample while the tunnel current $I_{\mathrm{t}}$ is measured. In most cases, the so-called constant-current mode is used in which the current is kept constant by adjusting the distance between tip and the sample. The image is then formed from the distance, i.e. tip height signal. Because of the exponential dependence of the tunnel current on the tip sample distance the technique is extremely sensitive with vertical resolution below $1 \AA$ A. Surface-adsorbed species such as single atoms and molecules can be imaged. The tunnel current is given by

$$
I_{\mathrm{t}} \propto \int_{0}^{\mathrm{eV}} \rho_{\mathrm{s}}\left(E_{\mathrm{F}}-e V+\epsilon\right) \cdot \rho_{\mathrm{t}}\left(E_{\mathrm{F}}+\epsilon\right)|M|^{2} \mathrm{~d} \epsilon,
$$

in which $\rho_{\mathrm{s}}$ and $\rho_{\mathrm{t}}$ are the sample and tip local densities of states (LDOS), $E_{\mathrm{F}}$ is the Fermi energy, $V$ the applied voltage and $M$ is the tunnel matrix element. Hence the STM images have to be interpreted as maps of electronic properties at the surface which do not have to coincide with a geometric topography. This is in particular important when imaging molecules which often have a LDOS at the Fermi energy much lower than a metal substrate, implying that the apparent height at which the molecules appear is less than the geometric height.

Among the many aspects of STM, there is the possibility of performing local spectroscopy of electronic, vibrational and magnetic states. Also, the option of working with spinpolarized tips is certainly of great interest when dealing with
SIMs on surfaces. It should also be mentioned here that spin-excitation spectroscopy (or inelastic electron tunneling spectroscopy) is able to reveal magnetic excitations of single surface-adsorbed atoms and molecules [150-156] such that this type of STM spectroscopy is certainly of high interest in view of the Ln SIMs discussed in this review.

\section{4. $\mathrm{LnPC}_{2}$}

In this Chapter, the surface studies on $\mathrm{TbPc}_{2}$ and relatives will be reviewed, loosely grouped by the substrates used.

5.4.1. Nonmagnetic metal surfaces. In a first effort, this SIM was deposited on a $\mathrm{Cu}\left(\begin{array}{lll}1 & 1 & 1\end{array}\right)$ surface using a printing technique [157]. It was found that the molecules exhibited a characteristic eight-lobed structure in STM images. Further, the study suggested that the $\mathrm{Tb}$ 4f states could indeed be accessed in STM experiments. In a subsequent X-ray study, Stepanow et al demonstrated by x-ray linear dichroism and XMCD that $\mathrm{TbPc}_{2}$ SIMs are oriented and exhibit strong magnetic anisotropy when deposited by thermal sublimation

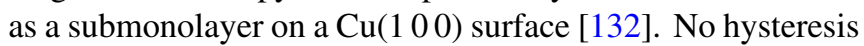
was observed which was attributed to the temperature of the experiment of $8 \mathrm{~K}$ and the data acquisition time of $20 \mathrm{~min}$ for a magnetization curve. Katoh and colleagues reported detailed STM addressing the Kondo effect in $\mathrm{TbPc}_{2}, \mathrm{DyPc}_{2}$ and $\mathrm{YPc}_{2}$ on $\mathrm{Au}(111)$ prepared by sublimation [131, 158, 159]. A mixture of the eight-lobed and four-lobed structures was observed, and the four-lobed structure was attributed to TbPc molecules, i.e. double-decker molecules which have lost one of the two ligands. It was further reported that the skew angle of the upper Pc ligand can be modified in STM experiments on single $\mathrm{TbPc}_{2}$ molecules on $\mathrm{Au}\left(\begin{array}{lll}1 & 1 & 1\end{array}\right)$ and $\operatorname{Ir}\left(\begin{array}{lll}1 & 1 & 1\end{array}\right)$ surfaces $[160,161]$. STM results were complemented by an XMCD study of a thick and a thin film of $\mathrm{TbPc}_{2}$ on

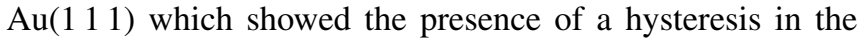
thick film, and in contrast only a small opening in the thin film deposit [162] (see figure 13). The structural integrity of $\mathrm{NdPc}_{2}$ molecules on different metal surfaces was investigated [163] revealing that on $\mathrm{Au}(111)$ the majority of molecules are of single-decker type or Pc only in agreement with the observations in [131]. Further work on $\mathrm{NdPc}_{2}$ deposited on $\mathrm{Cu}(100)$ addressed explicitly the issue of whether the $\mathrm{Nd}$ 4f states can be accessed in STM experiments [164]. The dynamic magnetic properties in the thin-film to bulk crossover regime were recently investigated in a muon-spin relaxation study [165], revealing that the magnetic fluctuations in the films are much stronger than in the bulk, which was attributed to differences in the packing of the molecules.

5.4.2. HOPG and graphene. An STM study of alkylfunctionalized $\mathrm{TbPc}_{2}$ molecules deposited on HOPG from the liquid phase revealed the formation of self-assembled checkerboard-type patches [166]. In a XMCD investigation, submonolayers and multilayers of the neutral and anionic $\mathrm{TbPc}_{2}$ were investigated [167] with samples prepared from solution. It was found that in the submonolayers both anionic and neutral molecules adsorb flat on the HOPG 

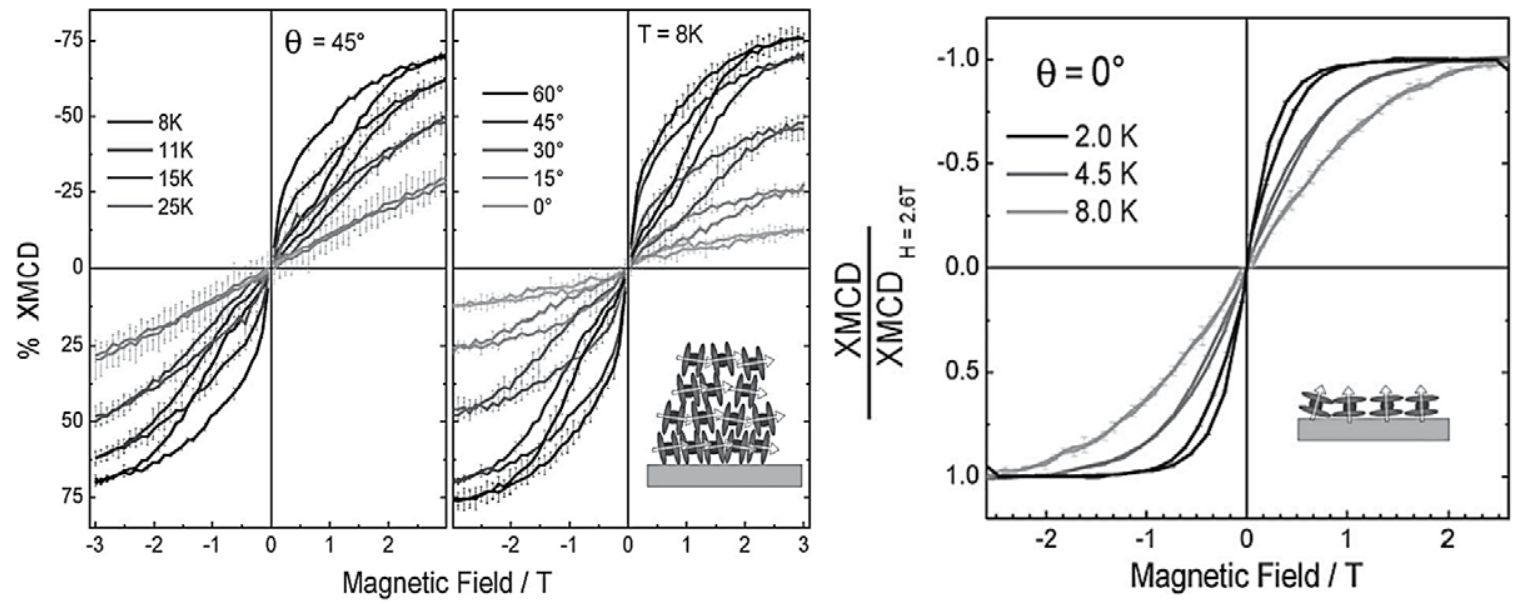

Figure 13. Magnetic hysteresis of a thick (left double panel) and a thin (right) film of $\mathrm{TbPc}_{2}$ on $\mathrm{Au}\left(\begin{array}{lll}1 & 1 & 1\end{array}\right)$. Reproduced with permission from [162].

surface. However, in the thick films the neutral molecules still exhibit orientation while there is an increased disorder for the anionic species. In a follow-up study Gonidec et al [168] reported a small hysteresis opening in a thick film as well as in a monolayer deposit. A similar experiment was carried out by Klar et al using UHV sublimation of the pristine neutral $\mathrm{TbPc}_{2}$ confirming the appearance of a hysteresis [169]. Moreover, the magnetic behavior of $\mathrm{DyPc}_{2}$ deposited on $\mathrm{HOPG}$ was studied, revealing a small hysteresis and remanence at sub-Kelvin temperatures. The magnetic properties of the protonated, neutral and anionic forms of the octaethylporphyrin- $\mathrm{Tb}^{\mathrm{III}}$ double decker molecules deposited on HOPG were investigated [107], and a derivative functionalized with long alkyl chains was studied. In [170] pyrene-functionalized $\mathrm{TbPc}_{2}$ molecules were deposited on a graphene nanoconstriction. The signature of the magnetic hysteresis could then be observed imprinted on the magnetoconducitivity of the device.

5.4.3. Oxides, semiconductors, ferromagnetic and antiferromagnetic metal surfaces. Several studies have been addressing the magnetic coupling of $\mathrm{TbPc}_{2}$ SIMs to ferromagnetic metals using XAS and XMCD, with partially non-congruent results. Lodi Rizzini et al demonstrated in a comprehensive study that $\mathrm{TbPc}_{2}$ shows magnetic exchange coupling to ferromagnetic substrates [171, 172] (see figure 14). The influence of different orientations of the substrate remanent magnetization (in-plane versus out-of-plane) was investigated as well as the effect of Li doping. In a further study by Klar et al [173] the coupling of $\mathrm{TbPc}_{2}$ to a Ni/Cu(1 00$)$ appeared to be significantly lower than in [171]. With the in-plane system $\mathrm{Co} / \mathrm{Cu}\left(\begin{array}{lll}1 & 0 & 0\end{array}\right)$ a very weak antiferromagnetic coupling was found, in contrast to a later study by Malavolti et al [174]. In that study $\mathrm{TbPc}_{2}$ molecules were also deposited on the ferromagnetic oxide $\mathrm{La}_{0.3} \mathrm{Sr}_{0.7} \mathrm{MnO}_{3}$ (LSMO), and no exchange coupling could be observed. It was found that the molecules adopt a standing geometry on LSMO, in contrast to the behaviour on noble metal surfaces. Moreover, in [175] the possibility of exchangebiasing SIMs was demonstrated using antiferromagnetic Mn thin films. In a spin-polarized STM study, spin-split molecular ligand orbitals could be observed directly on $\mathrm{TbPc}_{2}$ deposited on Co islands on $\operatorname{Ir}\left(\begin{array}{lll}1 & 1 & 1\end{array}\right)$ [176].

Besides oxide surfaces, semiconductors are the least explored substrates for the $\mathrm{TbPc}_{2}$ molecules. A recent study addresses this topic using a hydrogen-terminated $\operatorname{Si}\left(\begin{array}{lll}1 & 0 & 0\end{array}\right)$ surface [141]. The $\mathrm{TbPc}_{2}$ molecules were functionalized with long alkyl chains and chemically grafted onto the surface via the thermal hydrosilylation process. It was found that the molecules were partially oriented, and remarkably a hysteresis opening larger than in the bulk phase was observed in the monolayer.

5.4.4. Non-planar surfaces. Although the surfaces so far dealt with were planar, the grafting of $\mathrm{TbPc}_{2}$ molecules on to the non-planar surfaces of (single-walled) CNTs represents a directly related topic of as high interest. The underlying idea is to use the current passing through the nanotube to read out the magnetization state of single attached SIMs. An important fundamental aspect was the noncovalent grafting of SMMs and SIMs to the CNTs [177-179] such that isolated molecules can be attached without reducing the conductivity of the CNT. In consequence, pyrene-functionalized $\mathrm{TbPc}_{2}$ grafted on CNTs were used in a series of pioneering experiments demonstrating the feasibility of a supramolecular spin valve [24] as well as the observation of strong spin-phonon coupling between the CNT and a single attached molecule [25].

\section{5. $\operatorname{Ln}($ trensal)}

Out of the $\operatorname{Ln}$ (trensal) family $[58,108,110,111]$, the Er(trensal) SIM was studied in the monolayer regime on $\mathrm{Au}\left(\begin{array}{lll}1 & 1 & 1\end{array}\right)$ and $\mathrm{Ni} / \mathrm{Cu}\left(\begin{array}{lll}1 & 0 & 0\end{array}\right)$ surfaces [180]. The shape of the ligand is tripodal with the three legs being linked at an apex nitrogen atom as described in section 4.2. It has been shown that $\operatorname{Er}$ (trensal) can be sublimed in UHV and it was checked by x-ray photoelectron spectroscopy (XPS)

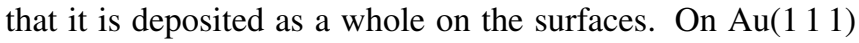
studies were performed on a multilayer and on a monolayer of molecules, which turned out to exhibit rather similar behavior, without a preferred orientation of the magnetic easy axis 
(a)
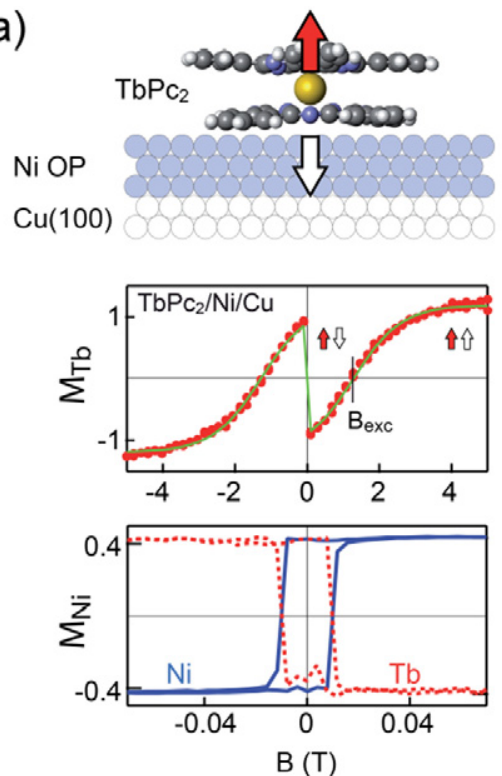

(b)
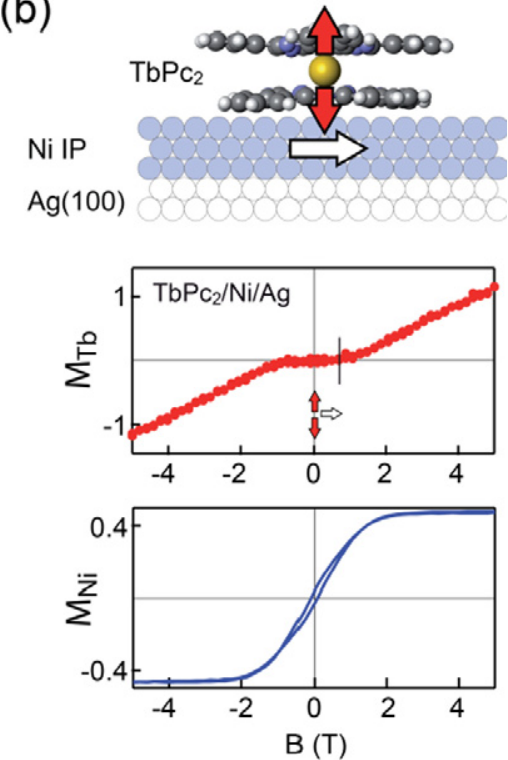

Figure 14. Element-specific magnetization from XMCD recorded on a submonolayer of $\mathrm{TbPc}_{2}$ deposited on ferromagnetic Ni thin films with $(a)$ out-of-plane and $(b)$ in-plane magnetic anisotropy. Reprinted figure with permission from [171]. Copyright (2011) by the American Physical Society.
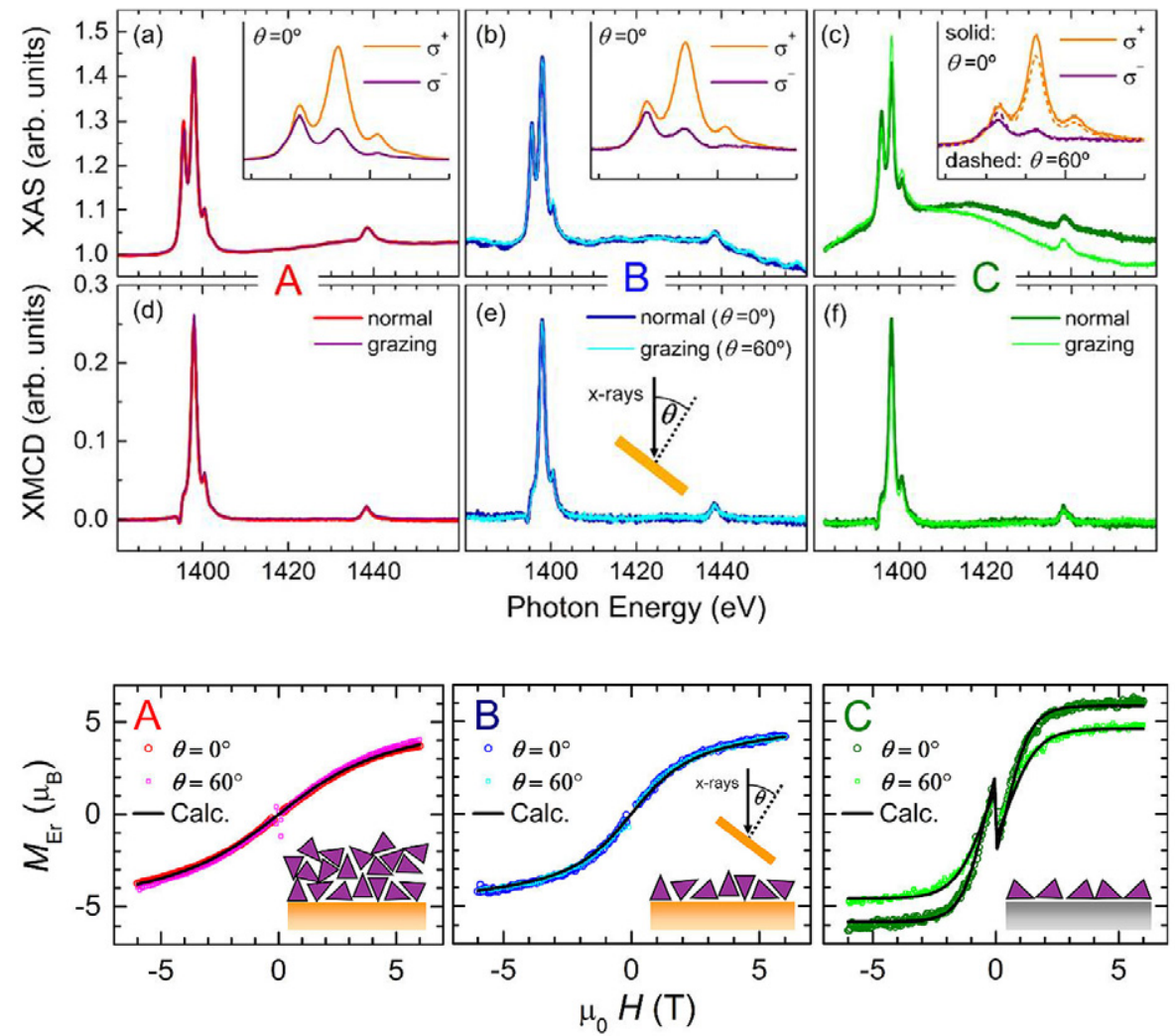

Figure 15. XAS and XMCD spectra recorded at the $\operatorname{Er~}_{4,5}$ edges and $\operatorname{Er} M(H)$ of (A) a multilayer of $\operatorname{Er}(\operatorname{trensal}) / \mathrm{Au}(111)$, (B) a

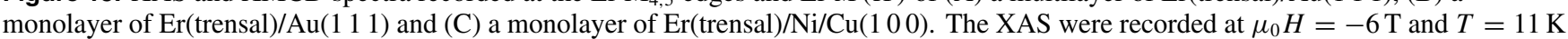
(A), $6 \mathrm{~K}$ (B) and $4 \mathrm{~K}$ (C). Adapted with permission from [180]. Copyright (2014) American Chemical Society.

(see figure 15). On a ferromagnetic Ni thin film grown on

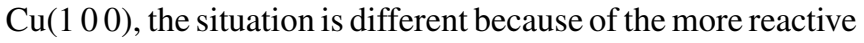
surface leading to hybridization between the molecules and the surface. This results in a preferential adsorption geometry of molecules lying on their sides trying to maximize interaction of their phenyl rings with the metal surface as suggested from DFT calculations. The molecules were shown to couple antiferromagnetically to the $\mathrm{Ni}$ substrate, as visible from the element-specific magnetization of sample $\mathrm{C}$ plotted in figure 15. The strength of the coupling is weak because of the three-dimensional nature of the molecules and the resulting long superexchange pathway as indicated by DFT calculations. 


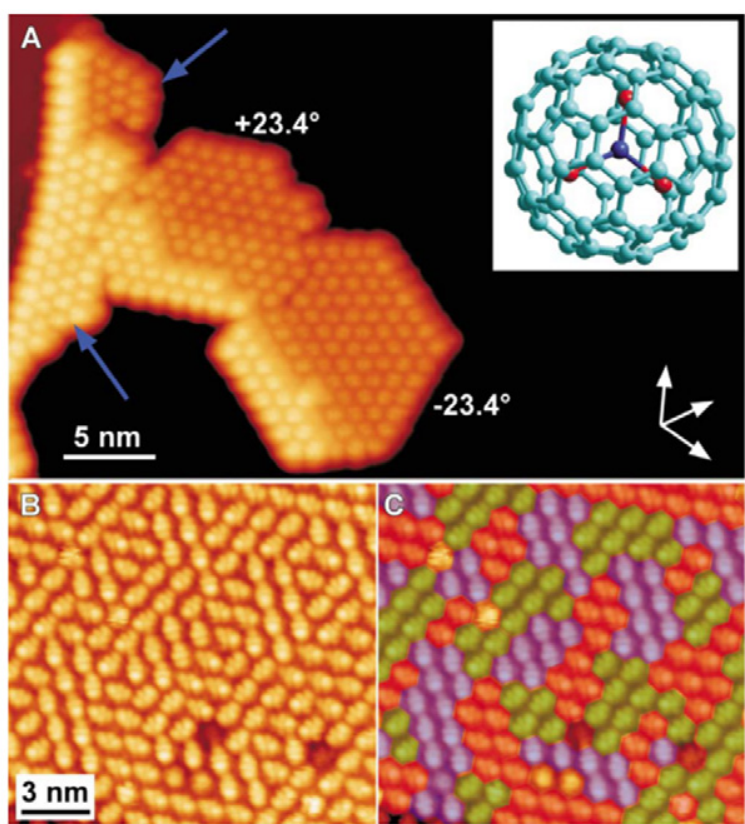

Figure 16. STM images showing $(A)$ islands of $\mathrm{Dy}_{3} \mathrm{~N} @ \mathrm{C}_{80}$ endohedral metallofullerenes on $\mathrm{Cu}(111),(B)$ formation of domains of differently oriented molecules and $(C)$ same as $(B)$, but with color-coded domains. Reprinted figure with permission from [118]. Copyright (2009) by the American Physical Society.

Up to now several studies have demonstrated that the electronic and magnetic structure of Ln SIMs is extremely sensitive to subtle perturbations of the ligand field $[58,94$,

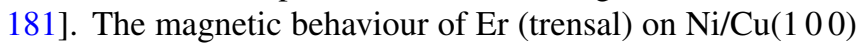
suggests that this plays a role also for surface-deposited SIMs, however, the small structural distortions are difficult to quantify experimentally in a submonolayer of surfaceadsorbed molecules.

\subsection{Endohedral metallofullerenes}

Endohedral metal-nitride fullerenes of type $\mathrm{M}_{x} \mathrm{Sc}_{3-x} \mathrm{~N} @ \mathrm{C}_{80}$ are thermally very stable and sublimable in UHV. Although reports on single-ion magnetic species adsorbed on surfaces are still lacking, experiments have been performed with the structurally similar polynuclear species $\mathrm{Dy}_{3} \mathrm{~N} @ \mathrm{C}_{80}$ [118], $\mathrm{Dy}_{2} \mathrm{ScN} @ \mathrm{C}_{80}$ [182] and $\mathrm{Gd}_{3} \mathrm{~N} @ \mathrm{C}_{80}$ [119]. Nevertheless, work on these species will be briefly reviewed here because their structure is closely related to the corresponding SIMs such as DySc $\mathrm{C}_{2} \mathrm{~N} @ \mathrm{C}_{80}$ [114]. The icosahedral $I_{\mathrm{h}}$ symmetry of the carbon cage and the rotational degrees of freedom of the endohedral unit give rise to a complex adsorption behavior. When deposited on $\mathrm{Cu}\left(\begin{array}{lll}1 & 1 & 1\end{array}\right)$, the $\mathrm{Dy}_{3} \mathrm{~N} @ \mathrm{C}_{80}$ molecules form differently ordered domains as shown in figure 16 [118]. It was found by an x-ray photoelectron diffraction experiment that the endohedral unit takes at least two different orientations, with the $\mathrm{N}$ atom resting at the center position of the cage. In $\mathrm{Dy}_{2} \mathrm{ScN} @ \mathrm{C}_{80}$ molecules deposited on $\mathrm{Rh}\left(\begin{array}{lll}1 & 1 & 1\end{array}\right)$ it was demonstrated by XAS and XMCD that the Dy(III) ions which carry the magnetic moment possess a net in-plane magnetic anisotropy implying that the plane of the endohedral unit must be parallel to the $\operatorname{Rh}\left(\begin{array}{lll}1 & 1 & 1\end{array}\right)$ surface [182]. Most interestingly, this compound exhibits a wide hysteresis with a sizeable opening at zero field. It was observed that the molecules in direct contact with the metal surface exhibit a smaller hysteresis opening than those in the multilayer. Remarkably, for the $\mathrm{Gd}_{3} \mathrm{~N} @ \mathrm{C}_{80}$ species magnetic exchange coupling of the endohedral unit through the $\mathrm{C}_{80}$ cage to a thin ferromagnetic Ni film [119] was observed.

\section{Future prospects}

While the theoretical foundations of the magnetism and electronic structure of lanthanides have been laid decades ago, these fundamental results appear under a new light in the age of molecular spintronics. Surface deposition experiments, elusive in earlier times, have become possible, and physicists and chemists alike work closely together to achieve challenging goals such as the creation of sophisticated devices.

Looking back to the recent years many promising results have been obtained in the relatively young field described in this review. However, there are many challenges ahead: Certainly on the wish list there are more sublimable molecules with long magnetization relaxation times. Moreover, sophisticated experimental and theoretical tools are needed to advance the understanding of changes of the molecular structure of surface-deposited species and the resulting changes of ligand field and/or magnetic properties. As an example the effect of the free conduction-band electrons present in a metal substrate on the magnetization relaxation times is not well understood yet and further work needs to be devoted to that topic. Abundant questions remain: What is the influence of oxide or semiconductor surfaces on the behavior of SIMs? Can information be written to or read from a single SIM on a planar surface on which SIMs could be organized, going beyond the pioneering experiments on SIMs anchored on carbon nanotubes? The interesting optical properties of some SIMs have not been further addressed yet apart from the study of absorption and luminescence spectra to understand the energy levels of SIMs in very few studies up to now. Studies reporting optical properties of surface-deposited SIMs are scarce $[183,184]$.

There is a great deal of experience from surface and interface studies of non-SIM mononuclear transition metal complexes as well as from research on molecular spin valves and tunneling devices. Hence the associated communities should be more strongly linked with those studying surfaceadsorbed SIMs, or in other words, the different involved fields should be brought closer together. If the relevant interactions and mechanisms are understood, they can possibly be controlled and harnessed in order to go beyond the current approach of using SIMs as independent building blocks that are put on surfaces, but rather the surface could be used to enhance the properties of SIMs.

\section{Acknowledgments}

The author is indebted to all collaborators and to the technical staff at the Ecole Polytechnique Federale de Lausanne and at 
the Swiss Light Source. Financial support by an Ambizione grant (no. PZ00P2_142474) of the Swiss National Science Foundation is gratefully acknowledged.

\section{References}

[1] Ishikawa N, Sugita M, Ishikawa T, Koshihara S and Kaizu Y 2003 Lanthanide double-decker complexes functioning as magnets at the single-molecular level J. Am. Chem. Soc. 125 8694-5

[2] Caneschi A, Gatteschi D, Sessoli R, Barra A L, Brunel L C and Guillot M 1991 Alternating current susceptibility, high field magnetization, and millimeter band EPR evidence for a ground $S=10$ state in

$\left[\mathrm{Mn}_{12} \mathrm{O}_{12}\left(\mathrm{CH}_{3} \mathrm{COO}\right)_{16}\left(\mathrm{H}_{2} \mathrm{O}\right)_{4}\right] \cdot 2 \mathrm{CH}_{3} \mathrm{COOH} \cdot 4 \mathrm{H}_{2} \mathrm{O}$ J. Am. Chem. Soc. 113 5873-4

[3] Sessoli R, Gatteschi D, Caneschi A and Novak M A 1993 Magnetic bistability in a metal-ion cluster Nature 365 141-3

[4] Gatteschi D, Sessoli R and Villain J 2006 Molecular Nanomagnets (Oxford: Oxford University Press)

[5] Freedman D E, Harman W H, Harris T D, Long G J, Chang C J and Long J R 2010 Slow magnetic relaxation in a high-spin iron(II) complex J. Am. Chem. Soc. 132 1224-5

[6] Zadrozny J M and Long J R 2011 Slow magnetic relaxation at zero field in the tetrahedral complex $[\mathrm{Co}(\mathrm{SPh}) 4]_{2}^{-} \mathrm{J}$. Am. Chem. Soc. 133 20732-4

[7] Sorace L, Benelli C and Gatteschi D 2011 Lanthanides in molecular magnetism: old tools in a new field Chem. Soc. Rev. 40 3092-104

[8] Leuenberger M N and Loss D 2001 Quantum computing in molecular magnets Nature 410 789-93

[9] Troiani F and Affronte M 2011 Molecular spins for quantum information technologies Chem. Soc. Rev. 40 3119-29

[10] Bertaina S, Gambarelli S, Mitra T, Tsukerblat B, Müller A and Barbara B 2010 Quantum oscillations in a molecular magnet Nature $\mathbf{4 6 6} 1006$

[11] Ardavan A, Rival O, Morton J J L, Blundell S J, Tyryshkin A M, Timco G A and Winpenny R E P 2007 Will spin-relaxation times in molecular magnets permit quantum information processing? Phys. Rev. Lett. 98057201

[12] Schlegel C, van Slageren J, Manoli M, Brechin E K and Dressel M 2008 Direct observation of quantum coherence in single-molecule magnets Phys. Rev. Lett. 101147203

[13] Martínez-Pérez M J et al 2012 Gd-based single-ion magnets with tunable magnetic anisotropy: molecular design of spin qubits Phys. Rev. Lett. 108247213

[14] Baldoví J J, Cardona-Serra S, Clemente-Juan J M, Coronado E, Gaita-Ariño A and Prima-García H 2013 Coherent manipulation of spin qubits based on polyoxometalates: the case of the single ion magnet $\left[\mathrm{GdW}_{30} \mathrm{P}_{5} \mathrm{O}_{110}\right]^{14-}$ Chem. Commun. 49 8922-4

[15] Thiele S, Balestro F, Ballou R, Klyatskaya S, Ruben M and Wernsdorfer W 2014 Electrically driven nuclear spin resonance in single-molecule magnets Science 344 1135-8

[16] Rocha A R, García-suárez V M, Bailey S W, Lambert C J, Ferrer J and Sanvito S 2005 Towards molecular spintronics Nat. Mater. 4 335-9

[17] Bogani L and Wernsdorfer W 2008 Molecular spintronics using single-molecule magnets Nat. Mater. 7 179-86

[18] Sanvito S 2011 Molecular spintronics Chem. Soc. Rev. $403336-55$

[19] Gatteschi D, Cornia A, Mannini M and Sessoli R 2009 Organizing and addressing magnetic molecules Inorg. Chem. 48 3408-19

[20] Domingo N, Bellido E and Ruiz-Molina D 2011 Advances on structuring, integration and magnetic characterization of molecular nanomagnets on surfaces and devices Chem. Soc. Rev. 41 258-302

[21] Cornia A, Mannini M, Sainctavit P and Sessoli R 2011 Chemical strategies and characterization tools for the organization of single molecule magnets on surfaces Chem. Soc. Rev. 40 3076-91

[22] Raman K V 2014 Interface-assisted molecular spintronics Appl. Phys. Rev. 1031101

[23] Cornia A and Mannini M 2014 Single-Molecule Magnets on Surfaces Structure and Bonding (Berlin: Springer) pp 1-38

[24] Urdampilleta M, Klyatskaya S, Cleuziou J-P, Ruben M and Wernsdorfer W 2011 Supramolecular spin valves Nat. Mater. 10 502-6

[25] Ganzhorn M, Klyatskaya S, Ruben M and Wernsdorfer W 2013 Strong spin-phonon coupling between a single-molecule magnet and a carbon nanotube nanoelectromechanical system Nat. Nanotechnol. 8 165-9

[26] Woodruff D N, Winpenny R E P and Layfield R A 2013 Lanthanide single-molecule magnets Chem. Rev. $1135110-48$

[27] Zhang P, Guo Y-N and Tang J 2013 Recent advances in dysprosium-based single molecule magnets: Structural overview and synthetic strategies Coord. Chem. Rev. 257 1728-63

[28] Jiang S-D, Wang B-W and Gao S 2014 Advances in lanthanide single-ion magnets Structure and Bonding (Berlin: Springer) pp 1-31

[29] Feltham H L C and Brooker S 2014 Review of purely 4f and mixed-metal nd-4f single-molecule magnets containing only one lanthanide ion Coord. Chem. Rev. 276 1-33

[30] Miyamachi T et al 2013 Stabilizing the magnetic moment of single holmium atoms by symmetry Nature $\mathbf{5 0 3} 242-6$

[31] Rau I G et al 2014 Reaching the magnetic anisotropy limit of a 3d metal atom Science 344 988-92

[32] Stevens K W H 1952 Matrix elements and operator equivalents connected with the magnetic properties of rare earth ions Proc. Phys. Soc. A $\mathbf{6 5} 209$

[33] Newman D J and Ng B 2000 Crystal Field Handbook (Cambridge: Cambridge University Press)

[34] Bauer E and Rotter M 2009 Magnetism of complex metallic alloys: crystalline electric field effects Properties and Applications of Complex Intermetallics (Book Series on Complex Metallic Alloys vol 2) (Singapore: World Scientific) pp 183-248

[35] Rinehart J D and Long J R 2011 Exploiting single-ion anisotropy in the design of f-element single-molecule magnets Chem. Sci. 2 2078-85

[36] Baldoví J J, Cardona-Serra S, Clemente-Juan J M, Coronado E, Gaita-Ariño A and Palii A 2012 Rational design of single-ion magnets and spin qubits based on mononuclear lanthanoid complexes Inorg. Chem. 51 12565-74

[37] Ishikawa N, Sugita M, Okubo T, Tanaka N, Iino T and Kaizu Y 2003 Determination of ligand-field parameters and f-electronic structures of double-decker bis(phthalocyaninato)lanthanide complexes Inorg. Chem. 42 2440-6

[38] AlDamen M A, Cardona-Serra S, Clemente-Juan J M, Coronado E, Gaita-ArinPo A, Martì̀-Gastaldo C, Luis F and Montero O 2009 Mononuclear lanthanide single molecule magnets based on the polyoxometalates $\left[\mathrm{Ln}\left(\mathrm{W}_{5} \mathrm{O}_{18}\right)_{2}\right]^{9-}$ and $\left[\mathrm{Ln}\left(\beta_{2}-\mathrm{SiW}_{11} \mathrm{O}_{39}\right)_{2}\right]^{13-}(\mathrm{LnIII}=\mathrm{Tb}$, Dy, Ho, Er, Tm, and Yb) Inorg. Chem. 48 3467-79

[39] Schäffer C E and Jørgensen C K 1965 The angular overlap model, an attempt to revive the ligand field approaches Mol. Phys. 9 401-12

[40] Urland W 1976 On the ligand-field potential for $f$ electrons in the angular overlap model Chem. Phys. 14 393-401 
[41] Urland W 1977 The application of the angular overlap model in the calculation of paramagnetic principal susceptibilities for fn-electron systems Chem. Phys. Lett. 46 457-60

[42] Kramers H A 1930 General theory of the paramagnetic rotation in crystals Proc. K. Akad. Van Wet. Te Amst. 33 959-72

[43] Abragam A and Bleaney B 1970 Electron Paramagnetic Resonance of Transition Ions (Oxford Classic Texts in the Physical Sciences) (Oxford: Oxford University Press)

[44] Chibotaru L F and Ungur L 2012 Ab initio calculation of anisotropic magnetic properties of complexes: I. Unique definition of pseudospin Hamiltonians and their derivation J. Chem. Phys. 137064112

[45] Luzon J, Bernot K, Hewitt I J, Anson C E, Powell A K and Sessoli R 2008 Spin chirality in a molecular dysprosium triangle: the archetype of the noncollinear ising model Phys. Rev. Lett. 100247205

[46] Dreiser J et al 2012 Direct observation of a ferri-to-ferromagnetic transition in a fluoride-bridged $3 \mathrm{~d}-4 \mathrm{f}$ molecular cluster Chem. Sci. 3 1024-32

[47] Furrer A 2010 Magnetic cluster excitations Int. J. Mod. Phys. B 24 3653-91

[48] Furrer A and Waldmann O 2013 Magnetic cluster excitations Rev. Mod. Phys. 85 367-420

[49] Urland W and Kremer R 1984 Electron spin resonance spectra of low-symmetry rare-earth complexes: tetraphenylarsonium pentakis(nitrato)ytterbate(III) Inorg. Chem. 23 1550-3

[50] Urland W, Kremer R and Furrer A 1986 Crystal field levels in tetraphenylarsoniumpentakis(nitrato)ytterbate(III) determined by neutron spectroscopy Chem. Phys. Lett. $132113-5$

[51] Hehlen M P and Güdel H U 1993 Optical spectroscopy of the dimer system $\mathrm{Cs}_{3} \mathrm{Yb}_{2} \mathrm{Br}_{9}$ J. Chem. Phys. 98 1768-75

[52] Barra A-L, Brunel L-C, Gatteschi D, Pardi L and Sessoli R 1998 High-frequency EPR spectroscopy of large metal ion clusters: from zero field splitting to quantum tunneling of the magnetization Acc. Chem. Res. 31 460-6

[53] van Slageren J et al 2003 Frequency-domain magnetic resonance spectroscopy of molecular magnetic materials Phys. Chem. Chem. Phys. 5 3837-43

[54] Hill S, Edwards R S, Jones S I, Dalal N S and North J M 2003 Definitive spectroscopic determination of the transverse interactions responsible for the magnetic quantum tunneling in $\mathrm{Mn}_{12}$-Acetate Phys. Rev. Lett. 90217204

[55] Gatteschi D, Barra A L, Caneschi A, Cornia A, Sessoli R and Sorace L 2006 EPR of molecular nanomagnets Coord. Chem. Rev. 250 1514-29

[56] Krzystek J, Ozarowski A and Telser J 2006 Multi-frequency, high-field EPR as a powerful tool to accurately determine zero-field splitting in high-spin transition metal coordination complexes Coord. Chem. Rev. $2502308-24$

[57] Schnegg A, Behrends J, Lips K, Bittl R and Holldack K 2009 Frequency domain Fourier transform THz-EPR on single molecule magnets using coherent synchrotron radiation Phys. Chem. Chem. Phys. 11 6820-5

[58] Pedersen K S et al 2014 Modifying the properties of $4 \mathrm{f}$ single-ion magnets by peripheral ligand functionalisation Chem. Sci. 5 1650-60

[59] Marx R et al 2014 Spectroscopic determination of crystal field splittings in lanthanide double deckers Chem. Sci. $53287-93$

[60] Reinhard C and Güdel H U 2002 High-resolution optical spectroscopy of $\mathrm{Na}_{3}\left[\mathrm{Ln}(\mathrm{dpa})_{3}\right] \cdot 13 \mathrm{H}_{2} \mathrm{O}$ with $\mathrm{Ln}=\mathrm{Er}^{3+}$, $\mathrm{Tm}^{3+}, \mathrm{Yb}^{3+}$ Inorg. Chem. 41 1048-55

[61] Paulovič J, Cimpoesu F, Ferbinteanu M and Hirao K 2004 Mechanism of ferromagnetic coupling in copper(II)-gadolinium(III) complexes J. Am. Chem. Soc. $1263321-31$
[62] Chibotaru L F, Ungur L and Soncini A 2008 The origin of nonmagnetic kramers doublets in the ground state of dysprosium triangles: evidence for a toroidal magnetic moment Angew. Chem. 120 4194-7

[63] Boulon M-E, Cucinotta G, Luzon J, Degl'Innocenti C, Perfetti M, Bernot K, Calvez G, Caneschi A and Sessoli R 2013 Magnetic anisotropy and spin-parity effect along the series of lanthanide complexes with DOTA Angew. Chem. Int. Ed. 52 350-4

[64] Singh S K, Gupta T and Rajaraman G 2014 Magnetic anisotropy and mechanism of magnetic relaxation in $\mathrm{Er}(\mathrm{III})$ single-ion magnets Inorg. Chem. 53 10835-45

[65] Aravena D and Ruiz E 2013 Shedding light on the single-molecule magnet behavior of mononuclear Dy III complexes Inorg. Chem. 52 13770-8

[66] Vieru V, Ungur L and Chibotaru L F 2013 Key role of frustration in suppression of magnetization blocking in single-molecule magnets J. Phys. Chem. Lett. 4 3565-9

[67] Schilder $\mathrm{H}$ and Lueken $\mathrm{H} 2004$ Computerized magnetic studies on $\mathrm{d}, \mathrm{f}, \mathrm{d}-\mathrm{d}, \mathrm{f}-\mathrm{f}$, and $\mathrm{d}-\mathrm{S}$, f-S systems under varying ligand and magnetic fields J. Magn. Magn. Mater. 281 17-26

[68] Speldrich M, Schilder H, Lueken H and Kögerler P 2011 A computational framework for magnetic polyoxometalates and molecular spin structures: CONDON 2.0 Isr. J. Chem. $51215-27$

[69] Van Leusen J, Speldrich M, Schilder H and Kögerler P 2015 Comprehensive insight into molecular magnetism via CONDON: full vs. effective models Coord. Chem. Rev. 289-290 137-48

[70] Baldoví J J, Clemente-Juan J M, Coronado E and Gaita-Ariño A 2014 Molecular anisotropy analysis of single-ion magnets using an effective electrostatic model Inorg. Chem. 53 11323-7

[71] Baldoví J J, Borrás-Almenar J J, Clemente-Juan J M, Coronado E and Gaita-Ariño A 2012 Modeling the properties of lanthanoid single-ion magnets using an effective point-charge approach Dalton Trans. 41 13705-10

[72] Baldoví J J, Cardona-Serra S, Clemente-Juan J M, Coronado E, Gaita-Ariño A and Palii A 2013 SIMPRE: a software package to calculate crystal field parameters, energy levels, and magnetic properties on mononuclear lanthanoid complexes based on charge distributions $J$. Comput. Chem. 34 1961-7

[73] Chilton N F, Collison D, McInnes E J L, Winpenny R E P and Soncini A 2013 An electrostatic model for the determination of magnetic anisotropy in dysprosium complexes Nat. Commun. 42551

[74] Van Vleck J H 1940 Paramagnetic relaxation times for titanium and chrome alum Phys. Rev. 57 426-47

[75] Orbach R 1961 Spin-lattice relaxation in rare-earth salts Proc. R. Soc. A 264 458-84

[76] Stevens K W H 1967 The theory of paramagnetic relaxation Rep. Prog. Phys. 30189

[77] Blagg R J, Ungur L, Tuna F, Speak J, Comar P, Collison D, Wernsdorfer W, McInnes E J L, Chibotaru L F and Winpenny R E P 2013 Magnetic relaxation pathways in lanthanide single-molecule magnets Nat. Chem. 5 673-8

[78] Guo Y-N, Xu G-F, Guo Y and Tang J 2011 Relaxation dynamics of dysprosium(III) single molecule magnets Dalton Trans. 40 9953-63

[79] Awschalom D D, DiVincenzo D P and Smyth J F 1992 Macroscopic quantum effects in nanometer-scale magnets Science 258 414-21

[80] Paulsen C and Park J-G 1995 Evidence for quantum tunneling of the magnetization in $\mathrm{Mn}_{12}$ Ac Quantum Tunneling of Magnetization (QTM '94 NATO ASI Series) ed L Gunther and B Barbara (Dordrecht: Springer) pp 189-207

[81] Friedman J R, Sarachik M P, Tejada J and Ziolo R 1996 Macroscopic measurement of resonant magnetization 
tunneling in high-spin molecules Phys. Rev. Lett. $763830-3$

[82] Thomas L, Lionti F, Ballou R, Gatteschi D, Sessoli R and Barbara B 1996 Macroscopic quantum tunnelling of magnetization in a single crystal of nanomagnets Nature 383 145-7

[83] Gatteschi D and Sessoli R 2003 Quantum tunneling of magnetization and related phenomena in molecular materials Angew. Chem. Int. Ed. 42 268-97

[84] Friedman J R and Sarachik M P 2010 Single-molecule nanomagnets Annu. Rev. Condens. Matter Phys. 1 109-28

[85] Ishikawa N, Sugita M and Wernsdorfer W 2005 Quantum tunneling of magnetization in lanthanide single-molecule magnets: bis(phthalocyaninato)terbium and bis(phthalocyaninato)dysprosium anions Angew. Chem. Int Ed. 44 2931-5

[86] Ishikawa N, Sugita M and Wernsdorfer W 2005 Nuclear spin driven quantum tunneling of magnetization in a new lanthanide single-molecule magnet: bis(phthalocyaninato)holmium anion J. Am. Chem. Soc. $1273650-1$

[87] Garanin D A and Chudnovsky E M 1997 Thermally activated resonant magnetization tunneling in molecular magnets: $\mathrm{Mn}_{12} \mathrm{Ac}$ and others Phys. Rev. B 56 11102-18

[88] Orbach R 1961 On the theory of spin-lattice relaxation in paramagnetic salts Proc. Phys. Soc. 77821

[89] Finn C B P, Orbach R and Wolf W P 1961 Spin-lattice relaxation in cerium magnesium nitrate at liquid helium temperature: a new process Proc. Phys. Soc. 77261

[90] Bierig R W, Weber M J and Warshaw S I 1964 Paramagnetic resonance and relaxation of trivalent rare-earth ions in calcium fluoride: II. Spin-lattice relaxation Phys. Rev. 134 A1504-16

[91] Orbach R and Blume M 1962 Spin-lattice relaxation in multilevel spin systems Phys. Rev. Lett. 8 478-80

[92] Walker M 1968 A T $^{5}$ spin-lattice relaxation rate for non-kramers ions Can. J. Phys. 46 1347-53

[93] Koike N, Uekusa H, Ohashi Y, Harnoode C, Kitamura F, Ohsaka T and Tokuda K 1996 Relationship between the skew angle and interplanar distance in four Bis(phthalocyaninato)lanthanide(III) tetrabutylammonium salts ([ $\left.\left.\mathrm{NBu}_{4}^{n}\right]\left[\mathrm{Ln}^{\mathrm{III}} \mathrm{Pc}_{2}\right] ; \mathrm{Ln}=\mathrm{Nd}, \mathrm{Gd}, \mathrm{Ho}, \mathrm{Lu}\right)$ Inorg. Chem. 35 5798-804

[94] Ishikawa N, Sugita M, Tanaka N, Ishikawa T, Koshihara S and Kaizu Y 2004 Upward temperature shift of the intrinsic phase lag of the magnetization of bis(phthalocyaninato)terbium by ligand oxidation creating an $S=1 / 2$ spin Inorg. Chem. 43 5498-500

[95] Gonidec M, Davies E S, McMaster J, Amabilino D B and Veciana J 2010 Probing the magnetic properties of three interconvertible redox states of a single-molecule magnet with magnetic circular dichroism spectroscopy $J$. Am. Chem. Soc. 132 1756-7

[96] Gonidec M, Krivokapic I, Vidal-Gancedo J, Davies E S, McMaster J, Gorun S M and Veciana J 2013 Highly reduced double-decker single-molecule magnets exhibiting slow magnetic relaxation Inorg. Chem. 52 4464-71

[97] Trojan K L, Hatfield W E, Kepler K D and Kirk M L 1991 Strong exchange coupling in lanthanide bis-(phthalocyaninato) sandwich compounds J. Appl. Phys. 69 6007-9

[98] Trojan K L, Kendall J L, Kepler K D and Hatfield W E 1992 Strong exchange coupling between the lanthanide ions and the phthalocyaninato ligand radical in bis(phthalocyaninato)lanthanide sandwich compounds Inorganica Chim. Acta 198-200 795-803

[99] Branzoli F, Carretta P, Filibian M, Zoppellaro G, Graf M J, Galan-Mascaros J R, Fuhr O, Brink S and Ruben M 2009 Spin dynamics in the negatively charged terbium (III) bis-phthalocyaninato complex J. Am. Chem. Soc. 131 4387-96

[100] Branzoli F, Filibian M, Carretta P, Klyatskaya S and Ruben M 2009 Spin dynamics in the neutral rare-earth single-molecule magnets $\left[\mathrm{TbPc}_{2}\right]^{0}$ and $\left[\mathrm{DyPc}_{2}\right]^{0}$ from $\mu \mathrm{SR}$ and NMR spectroscopies Phys. Rev. B 79220404

[101] Fukuda T, Shigeyoshi N, Yamamura T and Ishikawa N 2014 Magnetic relaxations arising from spin-phonon interactions in the nonthermally activated temperature range for a double-decker terbium phthalocyanine single molecule magnet Inorg. Chem. 53 9080-6

[102] Takamatsu S, Ishikawa T, Koshihara S and Ishikawa N 2007 Significant increase of the barrier energy for magnetization reversal of a single- $4 \mathrm{f}$-ionic single-molecule magnet by a longitudinal contraction of the coordination space Inorg. Chem. 46 7250-2

[103] Ishikawa N, Sugita M, Ishikawa T, Koshihara S and Kaizu Y 2004 Mononuclear lanthanide complexes with a long magnetization relaxation time at high temperatures: a new category of magnets at the single-molecular level J. Phys. Chem. B 108 11265-71

[104] Ishikawa N, Iino T and Kaizu Y 2002 Determination of ligand-field parameters and f-electronic structures of hetero-dinuclear phthalocyanine complexes with a diamagnetic yttrium(III) and a paramagnetic trivalent lanthanide ion J. Phys. Chem. A $1069543-50$

[105] Malavolti L, Mannini M, Car P-E, Campo G, Pineider F and Sessoli R 2013 Erratic magnetic hysteresis of $\mathrm{TbPc}_{2}$ molecular nanomagnets J. Mater. Chem. C $12935-42$

[106] Tanaka D, Inose T, Tanaka H, Lee S, Ishikawa $\mathrm{N}$ and Ogawa T 2012 Proton-induced switching of the single molecule magnetic properties of a porphyrin based $\mathrm{Tb}^{\mathrm{III}}$ double-decker complex Chem. Commun. 48 7796-8

[107] Inose T, Tanaka D, Tanaka H, Ivasenko O, Nagata T, Ohta Y, De Feyter S, Ishikawa N and Ogawa T 2014 Switching of single-molecule magnetic properties of $\mathrm{Tb}^{\mathrm{III}}$-porphyrin double-decker complexes and observation of their supramolecular structures on a carbon surface Chem.-Eur. J. $2011362-9$

[108] Flanagan B M, Bernhardt P V, Krausz E R, Lüthi S R and Riley M J 2001 Ligand-field analysis of an Er(III) complex with a heptadentate tripodal $\mathrm{N}_{4} \mathrm{O}_{3}$ ligand Inorg. Chem. 40 5401-7

[109] Kanesato M and Yokoyama T 1999 Synthesis and structural characterization of $\mathrm{Ln}(\mathrm{III})$ complexes $(\mathrm{Ln}=\mathrm{Eu}, \mathrm{Gd}, \mathrm{Tb}, \mathrm{Er}$, $\mathrm{Tm}, \mathrm{Lu}$ ) of tripodal tris[2-(salicylideneamino)ethyl]amine Chem. Lett. 28 137-8

[110] Flanagan B M, Bernhardt P V, Krausz E R, Lüthi S R and Riley M J 2002 A ligand-field analysis of the trensal $\left(\mathrm{H}_{3}\right.$ trensal $=2,2^{\prime}, 2^{\prime \prime}$-tris(salicylideneimino)triethylamine) ligand: an application of the angular overlap model to lanthanides Inorg. Chem. 41 5024-33

[111] Lucaccini E, Sorace L, Perfetti M, Costes J-P and Sessoli R 2014 Beyond the anisotropy barrier: slow relaxation of the magnetization in both easy-axis and easy-plane $\operatorname{Ln}$ (trensal) complexes Chem. Commun. 50 1648-51

[112] Stevenson S et al 1999 Small-bandgap endohedral metallofullerenes in high yield and purity Nature $40155-7$

[113] Popov A A, Yang S and Dunsch L 2013 Endohedral fullerenes Chem. Rev. 113 5989-6113

[114] Westerström R et al 2012 An endohedral single-molecule magnet with long relaxation times: $\mathrm{DySc}_{2} \mathrm{~N} @ \mathrm{C}_{80} J$. Am. Chem. Soc. 134 9840-3

[115] Westerström R et al 2014 Tunneling, remanence, and frustration in dysprosium-based endohedral single-molecule magnets Phys. Rev. B 89060406

[116] Dreiser J, Westerström R, Zhang Y, Popov A A, Dunsch L, Krämer K, Liu S-X, Decurtins S and Greber T 2014 
The metallofullerene field-induced single-ion magnet $\mathrm{HoSc}_{2} \mathrm{~N} @ \mathrm{C}_{80}$ Chem._Eur.J. 20 13536-40

[117] Zhang Y, Krylov D, Schiemenz S, Rosenkranz M, Westerström R, Dreiser J, Greber T, Büchner B and Popov A A 2014 Cluster-size dependent internal dynamics and magnetic anisotropy of $\mathrm{Ho}$ ions in $\mathrm{HoM}_{2} \mathrm{~N} @ \mathrm{C}_{80}$ and $\mathrm{Ho}_{2} \mathrm{MN} @ \mathrm{C}_{80}$ families $(\mathrm{M}=\mathrm{Sc}, \mathrm{Lu}, \mathrm{Y})$ Nanoscale 6 11431-8

[118] Treier M, Ruffieux P, Fasel R, Nolting F, Yang S, Dunsch L and Greber T 2009 Looking inside an endohedral fullerene: Inter- and intramolecular ordering of $\mathrm{Dy}_{3} \mathrm{~N} @ \mathrm{C}_{80}\left(I_{\mathrm{h}}\right)$ on Cu(111) Phys. Rev. B 80081403

[119] Hermanns C F et al 2013 Magnetic coupling of $\mathrm{Gd}_{3} \mathrm{~N} @ \mathrm{C}_{80}$ endohedral fullerenes to a substrate Phys. Rev. Lett. 111167203

[120] Cimpoesu F, Dragoe N, Ramanantoanina H, Urland W and Daul C 2014 The theoretical account of the ligand field bonding regime and magnetic anisotropy in the $\mathrm{DySc}_{2} \mathrm{~N} @ \mathrm{C}_{80}$ single ion magnet endohedral fullerene Phys. Chem. Chem. Phys. 16 11337-48

[121] AlDamen M A, Clemente-Juan J M, Coronado E, Martiì-Gastaldo C and Gaita-Ariño A 2008 Mononuclear lanthanide single-molecule magnets based on polyoxometalates J. Am. Chem. Soc. 130 8874-5

[122] Jiang S-D, Wang B-W, Sun H-L, Wang Z-M and Gao S 2011 An organometallic single-ion magnet J. Am. Chem. Soc 133 4730-3

[123] Jiang S-D, Liu S-S, Zhou L-N, Wang B-W, Wang Z-M and Gao S 2012 Series of lanthanide organometallic single-ion magnets Inorg. Chem. 51 3079-87

[124] Boulon M-E, Cucinotta G, Liu S-S, Jiang S-D, Ungur L, Chibotaru L F, Gao S and Sessoli R 2013 Angular-resolved magnetometry beyond triclinic crystals: out-of-equilibrium studies of $\mathrm{Cp} *$ ErCOT single-molecule magnet Chem.-Eur. J. 19 13726-31

[125] Jones P G, Hrib C G, Panda T K and Tamm M 2007 1,3-Diisopropylimidazolium bis(cyclooctatetraenyl)erbate(III) Acta Crystallogr. E 63 m2059-60

[126] Meihaus K R and Long J R 2013 Magnetic blocking at $10 \mathrm{~K}$ and a dipolar-mediated avalanche in salts of the bis $\left(\eta^{8}\right.$-cyclooctatetraenide) complex $\left[\mathrm{Er}(\mathrm{COT})_{2}\right]^{-} \mathrm{J} . \mathrm{Am}$. Chem. Soc. 135 17952-7

[127] Roy J J L, Korobkov I and Murugesu M 2014 A sandwich complex with axial symmetry for harnessing the anisotropy in a prolate erbium(III) ion Chem. Commun. 50 1602-4

[128] Ungur L, Le Roy J J, Korobkov I, Murugesu M and Chibotaru L F 2014 Fine-tuning the local symmetry to attain record blocking temperature and magnetic remanence in a single-ion magnet Angew. Chem. $1264502-6$

[129] Barth J V 2007 Molecular architectonic on metal surfaces Annu. Rev. Phys. Chem. 58 375-407

[130] Koepf M, Chérioux F, Wytko J A and Weiss J 2012 1D and 3D surface-assisted self-organization Coord. Chem. Rev. $2562872-92$

[131] Katoh K et al 2009 Direct observation of lanthanide(III)-phthalocyanine molecules on $\mathrm{Au}\left(\begin{array}{llll}1 & 1 & 1\end{array}\right)$ by using scanning tunneling microscopy and scanning tunneling spectroscopy and thin-film field-effect transistor properties of $\mathrm{Tb}(\mathrm{III})$ - and $\mathrm{Dy}(\mathrm{III})$-phthalocyanine molecules J. Am. Chem. Soc. 131 9967-76

[132] Stepanow S et al 2010 Spin and orbital magnetic moment anisotropies of monodispersed bis(phthalocyaninato)terbium on a copper surface $J$. Am. Chem. Soc. 132 11900-1

[133] Neddermeyer H 1996 Scanning tunnelling microscopy of semiconductor surfaces Rep. Prog. Phys. 59701
[134] Wolkow R A 1999 Controlled molecular adsorption on silicon: laying a foundation for molecular devices Annu. Rev. Phys. Chem. 50 413-41

[135] Hamers R J 2008 Formation and characterization of organic monolayers on semiconductor surfaces Annu. Rev. Anal. Chem. 1 707-36

[136] Diebold U 2003 The surface science of titanium dioxide Surf. Sci. Rep. 48 53-229

[137] Godlewski S and Szymonski M 2013 Adsorption and self-assembly of large polycyclic molecules on the surfaces of $\mathrm{TiO}_{2}$ single crystals Int. J. Mol. Sci. 14 2946-66

[138] Condorelli G G, Motta A, Pellegrino G, Cornia A, Gorini L, Fragalà I L, Sangregorio C and Sorace L 2008 Site-specific anchoring of tetrairon(III) single molecule magnets on functionalized $\operatorname{Si}(100)$ surfaces Chem. Mater. 20 2405-11

[139] Mannini M et al 2009 Magnetic memory of a single-molecule quantum magnet wired to a gold surface Nat. Mater. 8 194-7

[140] Corradini V, Ghirri A, del Pennino U, Biagi R, Milway V A, Timco G, Tuna F, Winpenny R E P and Affronte M 2010 Grafting molecular $\mathrm{Cr}_{7} \mathrm{Ni}$ rings on a gold surface Dalton Trans. 394928

[141] Mannini M et al 2014 Magnetic behaviour of $\mathrm{TbPc}_{2}$ single-molecule magnets chemically grafted on silicon surface Nat. Commun. 54582

[142] Stöhr J 1999 Exploring the microscopic origin of magnetic anisotropies with X-ray magnetic circular dichroism (XMCD) spectroscopy J. Magn. Magn. Mater. 200 470-97

[143] Funk T, Deb A, George S J, Wang H and Cramer S P 2005 $\mathrm{X}$-ray magnetic circular dichroism-a high energy probe of magnetic properties Coord. Chem. Rev. 249 3-30

[144] Van der Laan G and Figueroa A I 2014 X-ray magnetic circular dichroism - a versatile tool to study magnetism Coord. Chem. Rev. 277-278 95-129

[145] Thole B T, Carra P, Sette F and van der Laan G 1992 X-ray circular dichroism as a probe of orbital magnetization Phys. Rev. Lett. 68 1943-6

[146] Carra P, Thole B T, Altarelli M and Wang X 1993 X-ray circular dichroism and local magnetic fields Phys. Rev. Lett. 70 694-7

[147] Goedkoop J B, Thole B T, van der Laan G, Sawatzky G A, de Groot F M F and Fuggle J C 1988 Calculations of magnetic $\mathrm{x}$-ray dichroism in the $3 \mathrm{~d}$ absorption spectra of rare-earth compounds Phys. Rev. B 37 2086-93

[148] Piamonteze C et al 2012 X-Treme beamline at SLS: X-ray magnetic circular and linear dichroism at high field and low temperature J. Synchrotron Radiat. 19 661-74

[149] Ohresser P et al 2014 DEIMOS: a beamline dedicated to dichroism measurements in the $350-2500 \mathrm{eV}$ energy range Rev. Sci. Instrum. 85013106

[150] Heinrich A J, Gupta J A, Lutz C P and Eigler D M 2004 Single-atom spin-flip spectroscopy Science 306 466-9

[151] Chen X, Fu Y-S, Ji S-H, Zhang T, Cheng P, Ma X-C, Zou X-L, Duan W-H, Jia J-F and Xue Q-K 2008 Probing superexchange interaction in molecular magnets by spin-flip spectroscopy and microscopy Phys. Rev. Lett. 101197208

[152] Hirjibehedin C F, Lin C-Y, Otte A F, Ternes M, Lutz C P, Jones B A and Heinrich A J 2007 Large magnetic anisotropy of a single atomic spin embedded in a surface molecular network Science 317 1199-203

[153] Tsukahara N et al 2009 Adsorption-induced switching of magnetic anisotropy in a single iron(II) phthalocyanine molecule on an oxidized $\mathrm{Cu}\left(\begin{array}{lll}1 & 1 & 0\end{array}\right)$ surface Phys. Rev. Lett. 102167203

[154] Balashov T et al 2009 Magnetic anisotropy and magnetization dynamics of individual atoms and clusters of Fe and Co on Pt(1 11 1) Phys. Rev. Lett. 102257203

[155] Khajetoorians A A, Chilian B, Wiebe J, Schuwalow S, Lechermann F and Wiesendanger R 2010 Detecting 
excitation and magnetization of individual dopants in a semiconductor Nature 467 1084-7

[156] Brune H and Gambardella P 2013 Atomic and molecular magnets on surfaces Fundamentals of Picoscience (Boca Raton, FL: CRC Press) pp 447-70

[157] Vitali L, Fabris S, Conte A M, Brink S, Ruben M, Baroni S and Kern K 2008 Electronic structure of surface-supported bis(phthalocyaninato) terbium(III) single molecular magnets Nano Lett. 8 3364-8

[158] Katoh K, Komeda T and Yamashita M 2010 Surface morphologies, electronic structures, and Kondo effect of lanthanide(III)-phthalocyanine molecules on $\mathrm{Au}\left(\begin{array}{lll}1 & 1 & 1\end{array}\right)$ by using STM, STS and FET properties for next generation devices Dalton Trans. 39 4708-23

[159] Komeda T, Isshiki H, Liu J, Katoh K, Shirakata M, Breedlove B K and Yamashita M 2013 Variation of Kondo Peak observed in the assembly of heteroleptic 2,3-naphthalocyaninato phthalocyaninato $\mathrm{Tb}$ (III) double-decker complex on $\mathrm{Au}\left(\begin{array}{l}1 \\ 1\end{array} 1\right)$ ACS Nano 7 1092-9

[160] Komeda T, Isshiki H, Liu J, Zhang Y-F, Lorente N, Katoh K, Breedlove B K and Yamashita M 2011 Observation and electric current control of a local spin in a single-molecule magnet Nat. Commun. 2217

[161] Fu Y-S, Schwöbel J, Hla S-W, Dilullo A, Hoffmann G, Klyatskaya S, Ruben M and Wiesendanger R 2012 Reversible chiral switching of bis(phthalocyaninato) terbium(III) on a metal surface Nano Lett. 12 3931-5

[162] Margheriti L et al 2010 X-ray detected magnetic hysteresis of thermally evaporated terbium double-decker oriented films Adv. Mater. 22 5488-93

[163] Fahrendorf S, Matthes F, Bürgler D E, Schneider C M, Atodiresei N, Caciuc V, Blügel S, Besson C and Kögerler P 2014 Structural integrity of single bis(phthalocyaninato)-neodymium(III) molecules on metal surfaces with different reactivity SPIN 041440007

[164] Fahrendorf S, Atodiresei N, Besson C, Caciuc V, Matthes F, Blügel S, Kögerler P, Bürgler D E and Schneider C M 2013 Accessing 4f-states in single-molecule spintronics Nat. Commun. 42425

[165] Hofmann A, Salman Z, Mannini M, Amato A, Malavolti L, Morenzoni E, Prokscha T, Sessoli R and Suter A 2012 Depth-dependent spin dynamics in thin films of $\mathrm{TbPc}_{2}$ nanomagnets explored by low-energy implanted muons ACS Nano 6 8390-6

[166] Gómez-Segura J, Díez-Pérez I, Ishikawa N, Nakano M, Veciana J and Ruiz-Molina D 2006 2-D Self-assembly of the bis(phthalocyaninato)terbium(III) single-molecule magnet studied by scanning tunnelling microscopy Chem. Commun. 2866-8

[167] Biagi R et al 2010 X-ray absorption and magnetic circular dichroism investigation of bis(phthalocyaninato)terbium single-molecule magnets deposited on graphite Phys. Rev. B 82224406

[168] Gonidec M et al 2011 surface supramolecular organization of a terbium(III) double-decker complex on graphite and its single molecule magnet behavior J. Am. Chem. Soc. 133 6603-12

[169] Klar D, Candini A, Joly L, Klyatskaya S, Krumme B, Ohresser P, Kappler J-P, Ruben M and Wende H 2014 Hysteretic behaviour in a vacuum deposited submonolayer of single ion magnets Dalton Trans. 43 10686-9

[170] Candini A, Klyatskaya S, Ruben M, Wernsdorfer W and Affronte M 2011 Graphene spintronic devices with molecular nanomagnets Nano Lett. 11 2634-9

[171] Lodi Rizzini A et al 2011 Coupling single molecule magnets to ferromagnetic substrates Phys. Rev. Lett. 107177205

[172] Lodi Rizzini A et al 2014 Coupling of single, double, and triple-decker metal-phthalocyanine complexes to ferromagnetic and antiferromagnetic substrates Surf. Sci. $630361-74$

[173] Klar D et al 2013 Antiferromagnetic coupling of $\mathrm{TbPc}_{2}$ molecules to ultrathin $\mathrm{Ni}$ and Co films Beilstein J. Nanotechnol. 4 320-4

[174] Malavolti L et al 2013 Magnetism of $\mathrm{TbPc}_{2}$ SMMs on ferromagnetic electrodes used in organic spintronics Chem. Commun. 49 11506-8

[175] Lodi Rizzini A et al 2012 Exchange biasing single molecule magnets: coupling of $\mathrm{TbPc}_{2}$ to antiferromagnetic layers Nano Lett. 12 5703-7

[176] Schwöbel J, Fu Y, Brede J, Dilullo A, Hoffmann G, Klyatskaya S, Ruben M and Wiesendanger R 2012 Real-space observation of spin-split molecular orbitals of adsorbed single-molecule magnets Nat. Commun. 3953

[177] Giusti A et al 2009 Magnetic bistability of individual single-molecule magnets grafted on single-wall carbon nanotubes Angew. Chem. Int. Ed. 48 4949-52

[178] Bogani L, Danieli C, Biavardi E, Bendiab N, Barra A-L, Dalcanale E, Wernsdorfer W and Cornia A 2009 Single-molecule-magnet carbon-nanotube hybrids Angew. Chem. Int. Ed. $48746-50$

[179] Kyatskaya S, Mascarós J R G, Bogani L, Hennrich F, Kappes M, Wernsdorfer W and Ruben M 2009 Anchoring of rare-earth-based single-molecule magnets on single-walled carbon nanotubes J. Am. Chem. Soc. 131 15143-51

[180] Dreiser J et al 2014 Exchange interaction of strongly anisotropic tripodal erbium single-ion magnets with metallic surfaces ACS Nano 8 4662-71

[181] Cucinotta G, Perfetti M, Luzon J, Etienne M, Car P-E, Caneschi A, Calvez G, Bernot K and Sessoli R 2012 Magnetic anisotropy in a dysprosium/DOTA Single-molecule magnet: beyond simple magneto-structural correlations Angew. Chem. Int. Ed. 51 1606-10

[182] Westerström R et al 2015 Surface aligned magnetic moments and hysteresis of an endohedral single-molecule magnet on a metal Phys. Rev. Lett. 114087201

[183] Lopes M et al 2010 Surface-enhanced raman signal for terbium single-molecule magnets grafted on graphene ACS Nano 4 7531-7

[184] Robaschik P et al 2014 Optical properties and electrical transport of thin films of terbium(III) bis(phthalocyanine) on cobalt Beilstein J. Nanotechnol. 5 2070-8 